TITLE:

\title{
Pivotal role of liver sinusoidal endothelial cells in NAFLD/NASH progression.
}

\section{AUTHOR(S):}

Miyao, Masashi; Kotani, Hirokazu; Ishida, Tokiko; Kawai, Chihiro; Manabe, Sho; Abiru, Hitoshi; Tamaki, Keiji

\section{CITATION:}

Miyao, Masashi ...[et al]. Pivotal role of liver sinusoidal endothelial cells in NAFLD/NASH progression.. Laboratory investigation 2015, 95(10): 1130-1144

\section{ISSUE DATE:}

2015-10

URL:

http://hdl.handle.net/2433/202772

\section{RIGHT:}

This is the accepted manuscript of the article is available at http://dx.doi.org/10.1038/labinvest.2015.95. The full-tex file will be made open to the public on 27 July 2016 in accordance with publisher's 'Terms and Conditions for SelfArchiving'., この論文は出版社版でありません。引用の際には出版社版をご確認ご利用ください。; This is not the published version. Please cite only the published version. 
Pivotal role of liver sinusoidal endothelial cells in NAFLD/NASH progression

Masashi Miyao* M.D., Ph.D.; Hirokazu Kotani* „\# M.D., Ph.D.; Tokiko Ishida* M.D.;

Chihiro Kawai* B.S.; Sho Manabe* M.S.; Hitoshi Abiru* B.S.; and Keiji Tamaki* M.D., Ph.D.

*Department of Forensic Medicine, Kyoto University Graduate School of Medicine, Kyoto, Japan

\section{Corresponding author:}

\#Hirokazu Kotani,

Department of Forensic Medicine, Kyoto University Graduate School of Medicine,

Yoshida-Konoe-cho, Sakyo-ku, Kyoto 606-8501, Japan

Telephone: +81 75753 4474; Fax: +81 75761 9591;

E-mail: kotani@fp.med.kyoto-u.ac.jp

Sources of support: This work was supported by a Grant-in-Aid for Young Scientists

(B) (JSPS KAKENHI Grant Number 26860465) and a Kyoto University Young Scientists Start-up Grant (MM). 
Masashi Miyao 2

Running title: Liver sinusoidal endothelial injury in NAFLD 


\section{Abstract}

Liver sinusoidal endothelial cells (LSECs) are involved in the transport of nutrients, lipids, and lipoproteins, and LSEC injury occurs in various liver diseases including nonalcoholic fatty liver disease (NAFLD). However, the association between LSEC injury and NAFLD progression remains elusive. Accordingly, in this study, we aimed to elucidate the precise role of LSEC in the pathophysiology of NAFLD using two different mouse models, namely the choline-deficient, L-amino acid-defined (CDAA) and high fat diet (HFD) models. Administrations of these diets resulted in liver metabolic dysregulation mimicking human NAFLD, such as steatosis, ballooning, lobular inflammation, and fibrosis, as well as central obesity, insulin resistance, and hyperlipidemia. LSEC injury appeared from the simple steatosis phase, and preceded the appearance of activated Kupffer cells and hepatic stellate cells. These results indicate that LSEC injury may play a "gatekeeper" role in the progression from simple steatosis to the early nonalcoholic steatohepatitis (NASH) stage, and LSEC injury may be necessary for the activation of Kupffer cells and hepatic stellate cells, which in turn results in the development and perpetuation of chronic liver injuries. Taken together, our data provide new insights into the role of LSEC injury in NAFLD/NASH pathogenesis. 
Keywords: Capillarization; Choline-deficient L-amino acid-defined diet; Hepatic stellate cell; High fat diet; Kupffer cell; Macrophage

Abbreviations: ALP, alkaline phosphatase; ALT, aminotransferase; CD, cluster of differentiation; CDAA, choline-deficient, L-amino acid-defined; Col1 $\alpha 1$, collagen type I $\alpha 1$; Col4 $\alpha 1$, collagen type IV $\alpha$ 1; HFD, high-fat diet; HSC, hepatic stellate cell; IL-1 $\beta$, interleukin-1 $\beta$; IL-6, interleukin-6; LSEC, liver sinusoidal endothelial cell; MCP-1, monocyte chemotactic protein-1; NAFLD, nonalcoholic fatty liver disease; NASH, nonalcoholic steatohepatitis; SMA, smooth muscle actin; SEM, scanning electron microscopy; TEM, transmission electron microscopy; TIMP1, tissue inhibitor of metalloproteinase 1; TNF $\alpha$, tumor necrosis factor $\alpha$; VEGFR2, vascular endothelial growth factor receptor 2; VLDL, very low-density lipoprotein. 
Masashi Miyao 5

Nonalcoholic fatty liver disease (NAFLD) is one of the manifestations of metabolic syndrome, and its worldwide prevalence is continually increasing [1]. NAFLD ranges from simple steatosis, which follows a relatively benign course, to nonalcoholic steatohepatitis (NASH), which is a more severe form, and which can lead to fibrosis, cirrhosis, and, eventually, to hepatocellular carcinoma. Therefore, early diagnosis and management of NASH is important for improving the patient prognosis [2], and better understanding of NAFLD pathogenesis, particularly in the early stage, is thus essential for developing novel strategies for the diagnosis, prevention, and treatment of NAFLD.

Liver sinusoidal endothelial cells (LSECs) are the most abundant non-parenchymal cells in the liver and play important role in the transfer of nutrients, lipids, and lipoproteins. Under physiological conditions, LSECs are perforated by fenestrations and lack a basement membrane [3]; however, under pathological conditions, LSECs lose their fenestrations and form a continuous basement membrane [4]. This phenomenon is called "capillarization" as both these features are considered normal structures of the capillaries in other organs [5]. It has been demonstrated that sinusoidal capillarization precedes liver fibrosis in various liver diseases, including NAFLD [6-7], and our previous study using a cholangiopathy model also demonstrated that capillarization appeared prior to liver fibrosis [8]. Furthermore, Xie et al. recently reported that 
capillarization plays a pivotal role in hepatic stellate cell (HSC) activation and fibrogenesis during the late stage in a rat liver fibrosis model [9], and LSECs have been speculated to play an anti-inflammatory role in cooperation with Kupffer cells, and to play a pivotal role for fibrogenesis by promoting HSC activation [10-12]. However, the relationship between sinusoidal capillarization and NAFLD progression, and the interactions of LSECs with these cells in the different stages of NAFLD remain largely unknown.

In this study, we aimed to clarify the association between LSEC injury and NAFLD/NASH progression using the choline-deficient, L-amino acid-defined (CDAA) and high fat diet (HFD) models of NAFLD. In addition, we aimed to emphasize the importance of the interplay among LSECs, hepatocytes, Kupffer cells, and HSCs in order to expand our understanding of the pathogenesis of chronic liver diseases.

\section{MATERIALS AND METHODS}

\section{Animals}

Male C57BL/6 mice (CLEA Japan Inc., Tokyo, Japan) (8 weeks old, weighing 20-25 g) were used for all experiments. There is no single animal model of NAFLD completely reflecting the complexity of human disease [13]. Accordingly, it is important to use at 
least two separate animal models when studying this disorder. The CDAA diet is a well-established nutritional NAFLD model showing rapid and pronounced liver injury [14-15]. However, the metabolic profile of the model does not completely reflect all properties of NAFLD in terms of other metabolic dysregulations, because hepatic lipid accumulation of the model is mainly due to impaired secretion of very low-density lipoprotein (VLDL). Therefore, this model is considered suitable for evaluations of intrahepatic lesions in severe NASH. On the other hand, the HFD model more closely resembles the pathophysiology of human NAFLD with diabetes and central obesity. However, this model is requires more time and the hepatic lesions are less marked [13]. Thus, the HFD model is well suited for evaluations of capillarization in the early stages of NAFLD associated with diabetes and central obesity. In the CDAA mice, to induce steatohepatitis, the mice were fed a CDAA diet (Dyets Inc., Bethlehem, PA, USA) for 1, 4,8 , or 22 weeks. For the regression experiment, another group of animals was fed a CDAA diet for 8 weeks and allowed to recover on a standard diet for an additional 4 weeks. The HFD mice were fed an HFD (Dyets Inc.) for 8 and 22 weeks. The control mice were fed a standard diet.

The experimental animals were sacrificed at each indicated time point. Blood was collected by cardiac puncture after fasting for four hours. For light and electron 
microscopic analyses, the mice were perfused for 1 min with saline and fixed by transcardial perfusion (approximately $100 \mathrm{mmHg}$ ) with $10 \%$ buffered formaldehyde or $2 \%$ glutaraldehyde. At least three mice were treated and analyzed for each time period and treatment. All mice were housed in individual cages under specific pathogen-free conditions with food and water ad libitum. There was no difference in food intake between the groups (data not shown).

All experimental protocols were approved by the local Animal Care and Use Committee, and were performed according to the criteria outlined in the Guide for the Care and Use of Laboratory Animals prepared by the National Academy of Sciences, as published by the National Institutes of Health [16].

\section{Serum biochemical analysis}

The serum samples were stored at $-80^{\circ} \mathrm{C}$ until the analyses could be performed. The alanine aminotransferase (ALT), alkaline phosphatase (ALP), total-cholesterol, triglyceride, free fatty acids, total-bilirubin, and glucose levels were analyzed using a Hitachi 7180 analyzer (Hitachi, Tokyo, Japan).

\section{Histopathology}


Formalin-fixed paraffin-embedded sections were cut $4-\mu \mathrm{m}$ thick and stained with hematoxylin and eosin or Azan. Frozen sections were cut to $10 \mu \mathrm{m}$ and stained with Oil Red $\mathrm{O}$ for lipid analysis. A modified histological NAFLD/NASH score, as per the recommendations of the Nonalcoholic Steatohepatitis Clinical Research Network, was used [17]. Briefly, four histological features were semiquantitatively assessed: steatosis (0-3), lobular inflammation (0-3), hepatocellular ballooning (0-2), and fibrosis (0-4). The histological features of the specimens were independently assessed by an experienced pathologist (MM) and an attending pathologist (HK) in a blinded fashion, and a consensus diagnosis was obtained for each sample. To determine the interobserver reproducibility of each feature, unweighted kappa coefficients were calculated. The results showed good-to-excellent interobserver agreement, as demonstrated by a kappa coefficient of 0.72 (good) for steatosis, 0.82 (excellent) for lobular inflammation, 0.72 (good) for hepatocyte ballooning, and 0.77 (good) for fibrosis.

\section{Immunohistochemical studies}

Immunohistochemical staining of the specimens was performed according to the manufacturer's instructions. Briefly, antigen retrieval was carried out in a pressure cooker by boiling in $10 \mathrm{mM}$ citrate buffer ( $\mathrm{pH}$ 6.0), followed by washing with 
phosphate-buffered saline. Subsequently, endogenous peroxidase was quenched with $3 \% \mathrm{H}_{2} \mathrm{O}_{2}$ for $10 \mathrm{~min}$ at room temperature. After rinsing, the slides were treated overnight at $4{ }^{\circ} \mathrm{C}$ with a negative control reagent or the following optimally diluted primary antibodies: cluster of differentiation (CD) 31 (rabbit polyclonal; 1:50; Abcam, Cambridge, MA, USA), CD34 (rat monoclonal; 1:200; Abcam), F4/80 (rat monoclonal; 1:200; Abcam), and $\alpha$-smooth muscle actin ( $\alpha$ SMA; rabbit polyclonal; 1:400; Abcam). Next, the slides were incubated with anti-mouse and anti-rabbit horseradish peroxidase-conjugated secondary antibody (goat polyclonal; prediluted; MBL, Nagoya, Japan). Binding to the antibody was detected using the labeled polymer method. Diaminobenzidine was used as the chromogen, followed by counterstaining with hematoxylin. For quantitative assessment of protein expression, the staining of each immunohistochemical specimen was captured in 10 randomly selected fields at $200 \times$ magnification and calculated using ImageJ software (US National Institutes of Health, Bethesda, MD, USA).

\section{Quantitative reverse transcription polymerase chain reaction}

Total RNA was extracted from liver tissue using TRIzol (Invitrogen, Carlsbad, CA, USA). cDNA was synthesized from total RNA using SuperScript III reverse 
Masashi Miyao 11

transcriptase (Invitrogen). Real-time PCR was performed using FastStart SYBR Green Master (Roche Diagnostics, Basel, Switzerland) and Rotor-Gene Q (Qiagen, Venlo, Netherland). The primer pairs are shown in Supplementary Table 1. The relative target gene expressions were normalized to GAPDH mRNA expression.

\section{Transmission and scanning electron microscopic analyses}

Perfusion-fixed livers were cut into 1-mm sections for transmission electron microscopy (TEM) and into 2-mm sections for scanning electron microscopy (SEM). These sections were immersion fixed in $2 \%$ glutaraldehyde at $4{ }^{\circ} \mathrm{C}$ for 2 hours. The specimens for TEM were extensively washed with phosphate-buffered saline, postfixed in $1 \%$ osmium tetroxide, dehydrated in a graded series of ethanol, and embedded in Epon. Ultra-thin sections $(80 \mathrm{~nm})$ were cut on an Ultra microtome EM UC6 (Leica, Vienna, Austria), stained with $1 \%$ uranyl acetate, counterstained using the Reynolds method, and examined on an H-7650 electron microscope (Hitachi). The specimens for SEM were postfixed in $1 \%$ osmium tetroxide, dehydrated in a graded series of ethanol, and dried. Subsequently, the sections were coated with a thin layer of platinum/palladium and visualized under an S-4700 electron microscope (Hitachi). For evaluations of capillarization severity, the percent of open space area in the LSECs (porosity) was 
measured in 15 randomly selected fields at $10000 \times$ magnification on at least three animals per group, using ImageJ software.

\section{Statistical analysis}

Data are reported as the arithmetic means \pm SEM. All data were analyzed using SPSS version 20.0 software (SPSS, Tokyo, Japan). Statistical significance was determined using Student's $t$ test or the Mann-Whitney $U$ test with Bonferroni correction for multiple comparisons. For all analyses, a $P$-value $<0.05$ was considered statistically significant.

\section{RESULTS}

CDAA feeding induces macroscopical steatosis and hepatomegaly during the early phase of NAFLD/NASH pathogenesis

To determine the morphological changes occurring during the early phase of CDAA-induced NAFLD/NASH pathogenesis, we obtained tissue samples from the mice after up to 8 weeks of CDAA feeding. The CDAA-fed mice showed liver enlargement and pale yellow discoloring, indicating hepatic steatosis (Figure 1a). Although the body weight of the CDAA-fed mice was similar to that of the controls up 
to 8 weeks feeding, the liver weight/body weight ratio of the CDAA-fed mice was significantly increased from 4 weeks of feeding and onwards, indicating hepatic injury and hepatomegaly (Figure 1b). Moreover, the spleen weight/body weight ratio, an indicator of portal hypertension, was significantly increased after 8 weeks of the CDAA diet. Similarly, the visceral fat weight/body weight ratio was significantly increased in a time-dependent manner from 4 weeks of feeding, indicating that the CDAA-fed mice were progressing to central obesity. Next, we investigated the functional changes in CDAA-diet-induced NAFLD/NASH by serum biochemical analyses. To exclude the effects of aging, we compared the control 0 -week (8-week-old) with control 8-week (16-week-old) mice, and confirmed that there was no significant difference between the two control groups in all serum parameters (data not shown). Compared to the control mice, the CDAA-fed mice showed significant elevations of serum ALT and glucose from 1 week, and bilirubin and ALP levels from 4 weeks (Figure 1c). Interestingly, all CDAA-fed mice showed a significant reduction of the serum triglyceride levels, whereas no significant differences were observed in the serum cholesterol and free fatty acid levels; these findings may be explained by compensatory hepatic uptake of serum lipids or by impairment in VLDL secretion from the liver [18-19]. 
Masashi Miyao 14

CDAA feeding induces microscopical steatosis, intralobular inflammation, and pericellular fibrosis during the early to intermediate phase

To further evaluate the morphological changes in the early phase of the CDAA diet model, we performed histopathological examinations of the CDAA-fed mouse livers after up to 8 weeks of feeding. Both hematoxylin and eosin and Oil red $\mathrm{O}$ staining of the liver sections from the CDAA-fed mice revealed steatosis already after 1 week of CDAA feeding (Figure 2a and $\mathrm{b}$ ). At 1 week of CDAA feeding, no significant inflammatory infiltration or no significant fibrosis was found; thus, the mice at 1 week of CDAA feeding reflect simple steatosis, which represents a very early stage of NAFLD/NASH pathogenesis. After hepatic steatosis occurred, intralobular inflammation and pericellular fibrosis emerged from 4 weeks of CDAA feeding and were aggravated in the period leading up to 8 weeks of feeding, indicating that 4 weeks or more of CDAA feeding reflects the intermediate NASH stage (Figure 2a and $b$ ). Intriguingly, reticulin staining showed a different staining pattern between 4 and 8 weeks of CDAA feeding: the 4-week CDAA-fed mice showed a thick black staining pattern whereas the 8-week CDAA-fed mice showed a combination staining pattern of thick and pale black areas (Figure 2a). These different staining patterns may be explained by increased maturity of the collagen fibers at 8 weeks, because reticulin 
tends to stain immature collagen fibers (type III collagen) in thick black, whereas mature collagen fibers (type I collagen) stain pale black.

\section{Sinusoidal capillarization appears in the early phase of NAFLD/NASH pathogenesis}

To determine when capillarization appears in the NAFLD/NASH pathogenesis, we next investigated the LSEC in the CDAA-fed mice up to 8 weeks by SEM, TEM, and immunohistochemical analyses. In the control mice, the LSECs exhibited typical fenestrae grouped in sieve plates (Figure 3a). In contrast, all LSECs in the CDAA-fed mice were overtly defenestrated after up to 8 weeks of feeding, indicating capillarization (Figure 3a and Supplementary Figure 1). Quantitative assessment of the fenestrated area revealed that the CDAA diet caused approximately $20 \%, 8$-fold, and 28 -fold reductions in porosity after 1,4 , and 8 weeks of feeding, respectively (Figure 3b). In line with these findings, the expressions of the capillarization markers (CD31 and CD34) were significantly increased after 4 and 8 weeks CDAA feeding upon immunohistochemical analyses (Figure $3 \mathrm{c}$ and d).

Activated Kupffer cells and hepatic stellate cells appear after sinusoidal 
Masashi Miyao 16

\section{capillarization}

Next, we analyzed the timing of activation of the Kupffer cells and HSCs in the early phase of NAFLD/NASH. To achieve this, we assessed the morphological and functional changes of these cells by histological and immunohistochemical analyses. Because lipids are lost in formalin-fixed paraffin-embedded sections during tissue processing and because frozen tissue sample analysis has extremely low-resolution power, we first investigated toluidine blue staining semithin sections of resin-embedded specimens. The semithin sections revealed that lipogranulomas formed crown-like structures, unique histological features of Kupffer cells in NASH but not simple steatosis [20], in the intralobular regions after 4 weeks of CDAA feeding (Figure $4 \mathrm{a}$ ), which were exacerbated over time (data not shown). In the TEM analysis, quiescent Kupffer cells were seen in the control mice, while large and phagocytic macrophages with extending pseudopods were seen in the CDAA-fed mice after 4 weeks (Figure $4 \mathrm{~b}$ and Supplementary Figure 2). In line with these findings, the immunohistochemical analysis of F4/80, an activation maker of Kupffer cells, also showed increased intensity of the stained area after 4 weeks of CDAA feeding (Figure 4c). After 8 weeks of CDAA feeding, macrovesicular lipids encircled by F4/80-positive cells, indicating typical crown-like structures, were diffusely found throughout the liver (Figure 4c). Quiescent 
HSCs, "fat-storing cells," were seen in the control mice, whereas spindle-shaped collagen-producing cells, myofibroblasts, which may be derived from HSCs, were found in the space of Disse from 4 weeks of CDAA feeding (Figure $4 \mathrm{~b}$ and Supplementary Figure 2). Furthermore, immunohistochemical analysis of $\alpha \mathrm{SMA}$, an activation marker of HSCs, also displayed increased intensity of the stained area after 4 weeks of CDAA feeding (Figure 4c).

To further understand the mechanism of progression from simple steatosis to NASH, we next examined the gene expression changes in the early phase of NAFLD by quantitative RT-PCR analysis. The mRNA levels of inflammatory cytokines and chemokine such as tumor necrosis factor $\alpha(\mathrm{TNF} \alpha)$, interleukin-6 (IL-6), and monocyte chemotactic protein-1 (MCP-1) were significantly increased after 1 week of CDAA feeding, indicating that inflammatory responses and activating Kupffer cells had already emerged at the transcriptional level in the simple steatosis phase (Figure 4d). Moreover, the mRNA levels of vascular endothelial growth factor receptor 2 (VEGFR2) were significantly increased at 4 weeks, while the levels after 1 week of feeding tended to be increased, without statistical significance, indicating that LSEC regenerative changes appear from the simple steatosis phase to the early NASH phase. In addition, the mRNA levels of genes involved in HSC activation and fibrogenesis such as aSMA, tissue 
inhibitor of metalloproteinase 1 (TIMP1), interleukin-1 $\beta$ (IL-1 $)$ ), collagen type I $\alpha 1$ (Col1 $\alpha 1)$, and collagen type IV $\alpha 1(\mathrm{Col} 4 \alpha 1)$ were increased after 1 week of feeding, indicating that HSC activation and fibrotic responses emerge at the transcriptional level already in the simple steatosis phase (Figure $4 d$ ).

Taken together, our results suggest that capillarization is necessary for the appearance of activated Kupffer cells and HSCs, although activation of these cells at the transcriptional level is initiated already in the simple steatosis stage.

Sinusoidal capillarization is aggravated in the cirrhotic phase and shows partial recovery by discontinuation of the experimental diet

To determine the association between capillarization and disease progression in the late phase of the CDAA model, we performed macroscopical, microscopical, and serum analyses after 22 weeks of CDAA feeding. In both the macroscopic and histological analyses, the livers of the mice exhibited distinctly cirrhotic features, with or without hepatocellular adenoma (Figure 5a and Supplementary Figure 3). In line with these morphological findings, the serum ALT levels were not obviously increased, whereas the levels of ALP and bilirubin remained high, indicating that 22-week CDAA mice well reflect the symptoms and signs of the late NASH stage in humans (Table 1). 
To clarify whether capillarization worsens in the late phase of NAFLD/NASH, we next assessed LSEC injury in the 22-week CDAA mice. Significant decreases in porosity of approximately 57 -fold and 34-fold compared to the control mice at 0 and 22 weeks, respectively, were observed (Figure 5b and c). It should be noted that the LSEC fenestrae decrease can be, however, induced not only by pathologic stresses but also by aging, with this decrease in LSEC fenestrae with aging being referred to as pseudocapillarization [21]. As expected, although no significant difference in porosity was observed between the control 0-week and control 8-week mice (data not shown), the control 22-week (30-week-old) mice showed a significant decrease in porosity, indicating age-related pseudocapillarization (Figure 5b and c). However, this decrease in porosity of the control 22-week mice was negligible compared to that of the CDAA 22-week mice.

To determine whether capillarization and other pathogenic features could recover after withdrawal of CDAA feeding, mice fed for 8 weeks with the CDAA diet were returned to a standard diet for an additional 4 weeks and subsequently analyzed. The serum ALT, ALP, and bilirubin levels (Table 1) and all histological grading scores were almost completely normalized in these mice, whereas the staging of fibrosis partially remained, especially around the lipogranulomas (Figure 6a and b). Additionally, 
capillarization was also significantly recovered after 4 weeks of cessation of CDAA feeding; however, the degree of recovery was lower compared to that of the other pathological features (steatosis, inflammation, and hepatocyte ballooning) (Figure 6c and d), suggesting that the presence of capillarization upon SEM analysis has extremely high sensitivity and specificity in the NAFLD/NASH pathogenesis.

Prior studies have delineated that differentiated LSECs (fenestrated LSECs) promote HSC quiescence and vice versa $[9,22]$. Consequently, in the current study, to clarify the relationship between LSECs and HSCs, we next analyzed whether HSC quiescence occurred after 4 weeks cessation of CDAA feeding, when the LSECs have partially returned to a differentiated state. We found that the aSMA-positive cells (activated HSCs) had completely disappeared at that time point, and, instead, fat-storing quiescent HSCs appeared in the toluidine-stained semithin sections (Supplementary Figure 4).

These findings indicate that the state of differentiation of LSECs, which represent the severity of capillarization, correlates well with NAFLD/NASH disease progression and regression.

High fat feeding also shows steatosis with sinusoidal capillarization but without severe inflammation and fibrosis 
Masashi Miyao 21

In order to exclude that the results of capillarization are confounded by model-specific effects and to clarify the association between capillarization and hepatic steatosis, we conducted additional experiments using an HFD-fed mouse model, which shows severe steatosis but does not induce severe inflammatory and fibrotic changes until in the late phase. Macroscopically, both 8-week and 22-week HFD-fed mice showed hepatomegaly with pale yellow discoloring (Figure 7a and Table 1) and severe body weight and visceral fat weight gains (Table 1). Serum biochemical analyses showed significant increases in the cholesterol and glucose levels in both 8-week and 22-week HFD-fed mice, well reflecting human metabolic syndrome. Although the serum triglyceride level was decreased, this may again be explained by compensatory hepatic uptake of serum lipids or by impairment in the VLDL secretion from the liver [18-19]. The serum ALT level was increased only in the 22-week HFD-fed mice, whereas the serum ALP and bilirubin levels were not greatly increased. Histologically, the HFD 8-week mice showed hepatic steatosis but no inflammatory and fibrotic changes compared to the controls. The HFD 22-week mice showed severe hepatic steatosis and significant, but not severe, inflammatory and fibrotic changes (Figure 7b-d). These results suggest that the HFD 8 -week feeding mouse model mimics the human simple steatosis stage whereas the HFD 22-week feeding mice mimic the early NASH stage. 
We next evaluated whether capillarization occurs in HFD-fed mice using SEM and TEM analyses. Capillarization was not detected in HFD 8-week mice but was severe in the HFD 22-week mice, as compared to each control (Figure 7e, f and Supplementary Figure 5). The severity of capillarization in the HFD 22-week mice was less prominent than that of the CDAA 22-week mice.

Taken together, these results indicate that capillarization may play a "gatekeeper" role in the progression from simple steatosis to the early NASH stage, and may represent a generalized change in NAFLD/NASH pathogenesis.

Finally, to determine when intracellular organelle injuries of hepatocyte appear in the NAFLD pathogenesis, we analyzed the endoplasmic reticula (ERs), mitochondria, autophagosomes and lysosomes, nuclei, and bile canaliculi in both models by TEM. In the CDAA mice, enlarged ERs first appeared from 4 weeks of feeding (Supplementary Figure 6). Deformed mitochondria and an increase of autophagosomes and lysosomes appeared from 8 weeks of feeding, while pseudoinclusion bodies in the nuclei and loss of microvilli of bile canaliculi were identified at 22 weeks of feeding. In HFD mice, deformed mitochondria first appeared from 8 weeks of feeding. Enlarged ERs, an increase of autophagosomes and lysosomes, and pseudoinclusion bodies in the nuclei were detected at 22 weeks of feeding (Supplementary Figure 7). No pathological 
changes were detected in the bile canaliculi of HFD-fed mice.

\section{DISCUSSION}

Capillarization has been known to occur in many different kinds of liver injuries (e.g., cirrhosis, hepatitis, alcoholic liver injury, and arsenic poisoning), to influence liver regeneration processes, and to naturally increase in severity with age (pseudocapillarization); however, the relationship between capillarization and NAFLD remains elusive [6, 23-24]. To our knowledge, our comprehensive macroscopical, microscopical, ultrastructural, biochemical, and quantitative RT-PCR analyses of two distinct NAFLD models are the first to reveal that capillarization arises during the progression from simple steatosis to the early NASH stage, and that it worsens in a time-dependent manner up until the late stage (Figures 3 and 7). Furthermore, we here revealed that the relationship of capillarization with disease progression is not model-specific, suggesting that capillarization is a generalized change of NAFLD/NASH pathogenesis. These new kinetic understandings of capillarization in the NAFLD progression may provide more information for future research efforts on NAFLD. Especially, focusing on capillarization may help improve the understanding of the initiating mechanisms of NASH in the early stages, and may eventually lead to the 
discovery of novel strategies for the prevention of disease progression and for promotion of disease regression in the late stages.

Kupffer cells and HSCs have been known to play critical roles in triggering fibrosis via monocyte recruitment and excessive collagen production [25]. However, the interactions of LSECs with Kupffer cells and HSCs in NAFLD pathogenesis remain largely unknown [26]. The current study examined the morphological and functional changes of these cells, and showed that both types of cells appeared activated after capillarization. In the CDAA model, immunohistochemical analysis of the macrophage activation maker F4/80 revealed that the activated Kupffer cells appeared from the early NASH phase, and were identified as typical crown-like structures from the intermediate NASH phase (Figure 4c). HSCs, differentiated lipid-storing cells found in the space of Disse, were identified in the control and simple steatosis phase (CDAA 1-week and HFD 8-week mice), but were not observed after the early NASH phase (CDAA 4-week and HFD 22-week mice). Meanwhile, from the early NASH phase, instead of differentiated HSCs, many dedifferentiated HSCs, spindle-shaped aSMA-positive cells, appeared around the necrotic hepatocytes and were found to release excessive collagen fibers (Figure 4b, c, and Supplementary Figure 4). In accordance with the observed HSC dedifferentiation, pericellular fibrosis appeared from the early phase, and 
progressed to cirrhosis in the late phase (Figure 5 and Supplementary Figure 3). Moreover, the quantitative RT-PCR analysis showed that the mRNA levels involved in inflammation and Kupffer cell activation, vascular regeneration, and fibrogenesis and HSC activation increased already after 1 week of CDAA feeding, indicating that these changes were initiated at the transcriptional level already in the simple steatosis phase (Figure 4d). Collectively, these results suggest that capillarization is the earliest morphological change in NASH pathogenesis, and that it plays critical roles in hepatic inflammation, fibrosis, cirrhosis, and hepatocellular carcinoma development through activation of Kupffer cells and HSCs.

In the current study, we found that various common intracellular organelle injuries of hepatocytes appeared after capillarization in both models. First, enlarged ERs, deformed mitochondria, and an increase of autophagosomes and lysosomes appeared, after which pseudoinclusion bodies in the nuclei and loss of microvilli in the bile canaliculi were recognized (Supplementary Figure 6 and 7). These results suggest that capillarization due to excessive hepatic lipid accumulation and elevated circulating lipids may lead to endoplasmic reticulum stress, mitochondrial dysfunction, cytoskeleton alterations, and DNA damages in the hepatocytes. Further studies are needed to determine whether sinusoidal capillarization is responsible for these 
hepatocyte organelle injuries in the NAFLD progression.

One obvious advantage of diet-induced NAFLD models is the possibility of performing regression experiments. In this study, we evaluated the restoration levels of individual features of NAFLD following cessation of CDAA administration. Macroscopically, CDAA cessation resulted in almost complete recovery in terms of body weight and visceral fat; however, the spleen weight (an indicator of portal hypertension) remained high (Table 1). Moreover, similar to the results of the macroscopical analyses, the microscopical findings of NASH, such as hepatic steatosis, intralobular inflammation, and hepatocyte ballooning, were also recovered (Figure 6a and b). On the other hand, slight pericellular fibrosis remained around the dead hepatocytes, with crystallized lipid droplets, and the restoration of capillarization showed only a $40 \%$ recovery in porosity (Figure $6 \mathrm{c}$ and d). As capillarization and pericellular fibrosis are known to be closely related to portal hypertension, our results suggest that sinusoidal capillarization is a delaying factor for the healing processes of fibrosis, and is a preserving factor of portal hypertension in NAFLD [27-28].

In this study, we provided the first evidence showing that capillarization may play a "gatekeeper" role in the progression from simple steatosis to the early NASH stage. Capillarization may occur prior to overt inflammation in the early stage, and plays a role 
in fibrogenesis in the late stage as well as delays the healing processes of portal hypertension in the recovering stage. Based on our findings herein, we propose the following mechanisms of NAFLD/NASH progression: 1) hepatic steatosis with proinflammatory and profibrogenic cytokine release occurs due to excessive fat intake or as a result of metabolic dysregulation or genetic susceptibility; consequently, 2) capillarization appears, followed by 3) definite inflammatory cell infiltrations, 4) pericellular fibrosis, and, eventually, 5) cirrhosis and hepatocellular carcinoma.

There are several clinical implications of the findings reported here. First, assessing the severity of LSEC injuries could help distinguish between simple steatosis and the early NASH stage, potentially leading to earlier detection of NASH. Second, development of drugs that can maintain a differentiated state for LSECs may prevent progression and promote recovery of NAFLD/NASH. Third, drugs that influence the differentiation state of LSECs may also be applied to the prevention and treatment of systemic vascular diseases, especially for atherosclerosis [29], as LSEC injury and dyslipidemia secondary to impaired lipid transport by LSEC injury might indirectly pertain to the capillary vessels of other organs such as the heart, lungs, and kidneys [3, 30].

A limitation of the present study is that, although we clarified that sinusoidal 
capillarization correlates well with NAFLD/NASH progression, we did not determine to what degree capillarization contributes to NAFLD/NASH progression. Further studies, for example focusing on the effects of administrating drugs that influence the restoration and capillarization of the LSEC phenotype, could resolve this question.

In conclusion, the results obtained herein provide new insights into NAFLD/NASH pathogenesis. LSEC injuries in the early stage of NAFLD appear to be necessary for the activation of Kupffer cells and HSCs, which in turn results in the development and perpetuation of chronic liver injuries. This insight may help to elucidate the crosstalk among hepatocytes, Kupffer cells, HSCs, and LSECs in NAFLD/NASH progression, and to help establish novel strategies for early diagnosis, prevention, and treatment in chronic liver diseases. 


\section{ACKNOWLEDGEMENTS}

This work was supported by a Grant-in-Aid for Young Scientists (B) (JSPS KAKENHI

Grant Number 26860465) and a Kyoto University Young Scientists Start-up Grant $(\mathrm{MM})$.

We gratefully acknowledge Haruyasu Kohda and Keiko Furuta for their skillful technical assistance with the electron microscopy. Further, we thank Dr. Leila Jemail and Editage for their careful reading of the manuscript and their thoughtful comments. We also acknowledge Hirozo Minami for his technical assistance with the histopathological analyses.

\section{DISCLOSURE/CONFLICT OF INTEREST}

The authors declare no conflict of interest.

\section{SUPPLEMENTARY INFORMATION}

Supplementary information is available at Laboratory Investigation's website. 


\section{References}

[1] Chalasani N, Younossi Z, Lavine JE, et al. The diagnosis and management of non-alcoholic fatty liver disease: practice guideline by the American Gastroenterological Association, American Association for the Study of Liver Diseases, and American College of Gastroenterology. Gastroenterology 2012;142:1592-1609.

[2] Gaidos JK, Hillner BE, Sanyal AJ. A decision analysis study of the value of a liver biopsy in nonalcoholic steatohepatitis. Liver Int 2008;28:650-658.

[3] Wisse E, De Zanger RB, Charels K, et al. The liver sieve: considerations concerning the structure and function of endothelial fenestrae, the sinusoidal wall and the space of Disse. Hepatology 1985;5:683-692.

[4] Braet F, Wisse E. Structural and functional aspects of liver sinusoidal endothelial cell fenestrae: a review. Comp Hepatol 2002;1:1.

[5] Schaffner F, Poper H. Capillarization of hepatic sinusoids in man. Gastroenterology $1963 ; 44: 239-242$.

[6] Xie G, Choi SS, Syn WK, et al. Hedgehog signalling regulates liver sinusoidal endothelial cell capillarisation. Gut 2013;62:299-309.

[7] Lee YA, Wallace MC, Friedman SL. Pathobiology of liver fibrosis: a translational success story. Gut 2015;64:830-841. 
[8] Miyao M, Ozeki M, Abiru H, et al. Bile canalicular abnormalities in the early phase of a mouse model of sclerosing cholangitis. Dig Liver Dis 2013;45:216-225.

[9] Xie G, Wang X, Wang L, et al. Role of differentiation of liver sinusoidal endothelial cells in progression and regression of hepatic fibrosis in rats. Gastroenterology 2012;142:918-927.e6.

[10] Brock RW, Dorman RB. Obesity, insulin resistance and hepatic perfusion. Microcirculation 2007;14:339-347.

[11] Miller AM, Wang H, Park O, et al. Anti-inflammatory and anti-apoptotic roles of endothelial cell STAT3 in alcoholic liver injury. Alcohol Clin Exp Res 2010;34:719-725.

[12] Leclercq IA, Da Silva Morais A, Schroyen B, et al. Insulin resistence in hepatocytes and sinusoidal liver cells: mechanisms and consequences. J Hepatol 2007;47:142-156.

[13] Takahashi Y, Soejima Y, Fukusato T. Animal models of nonalcoholic fatty liver disease/nonalcoholic steatohepatitis. World J Gastroenterol 2012;18:2300-2308. 
[14] Denda A, Kitayama W, Kishida H, et al. Development of hepatocellular adenomas and carcinomas associated with fibrosis in C57BL/6J male mice given a choline-deficient, L-amino acid-defined diet. Jpn J Cancer Res 2002;93:125-132.

[15] Caballero F, Fernández A, Matías N, et al. Specific contribution of methionine and choline in nutritional nonalcoholic steatohepatitis: impact on mitochondrial S-adenosyl-L-methionine and glutathione. J Biol Chem 2010;285:18528-18536.

[16] NIH. Guide for the Care and Use of Laboratory Animals. National Academy Press: National Academy of Science, 1996, ISBN: 0-309-05377-3.

[17] Kleiner DE, Brunt EM, Van Natta M, et al. Design and validation of a histological scoring system for nonalcoholic fatty liver disease. Hepatology 2005;41:1313-1321.

[18] Stanković MN, Mladenović DR, Duričić I, et al. Time-dependent changes and association between liver free fatty acids, serum lipid profile and histological features in mice model of nonalcoholic fatty liver disease. Arch Med Res 2014;45:116-124.

[19] Clapper JR, Hendricks MD, Gu G, et al. Diet-induced mouse model of fatty liver disease and nonalcoholic steatohepatitis reflecting clinical disease progression and methods of assessment. Am J Physiol Gastrointest Liver Physiol 2013;305:G483-G495. [20] Ioannou GN, Haigh WG, Thorning D, et al. Hepatic cholesterol crystals and crown-like structures distinguish NASH from simple steatosis. $J$ Lipid Res 
2013;54:1326-1334.

[21] Le Couteur DG, Cogger VC, Markus AM, et al. Pseudocapillarization and associated energy limitation in the aged rat liver. Hepatology 2001;33:537-543.

[22] Deleve LD, Wang X, Guo Y. Sinusoidal endothelial cells prevent rat stellate cell activation and promote reversion to quiescence. Hepatology 2008;48:920-930.

[23] DeLeve LD. Liver sinusoidal endothelial cells and liver regeneration. J Clin Invest 2013;123:1861-1866.

[24] Furrer K, Rickenbacher A, Tian Y, et al. Serotonin reverts age-related capillarization and failure of regeneration in the liver through a VEGF-dependent pathway. Proc Natl Acad Sci U S A 2011;108:2945-2950.

[25] Tosello-Trampont AC, Landes SG, Nguyen V, et al. Kupffer cells trigger nonalcoholic steatohepatitis development in diet-induced mouse model through tumor necrosis factor-alpha production. J Biol Chem 2012;287:40161-40172.

[26] Pasarín M, La Mura V, Gracia-Sancho J, et al. Sinusoidal endothelial dysfunction precedes inflammation and fibrosis in a model of NAFLD. PLoS One 2012;7:e32785.

[27] DeLeve LD, Wang X, Hu L, et al. Rat liver sinusoidal endothelial cell phenotype is maintained by paracrine and autocrine regulation. Am J Physiol Gastrointest Liver Physiol 2004;287:G757-G763. 
[28] Dill MT, Rothweiler S, Djonov V, et al. Disruption of Notch1 induces vascular remodeling, intussusceptive angiogenesis, and angiosarcomas in livers of mice. Gastroenterology 2012;142:967-977.e2.

[29] Le Couteur DG, Fraser R, Cogger VC, et al. Hepatic pseudocapillarisation and atherosclerosis in ageing. Lancet 2002;359:1612-1615.

[30] Fraser R, Dobbs BR, Rogers GW. Lipoproteins and the liver sieve: the role of the fenestrated sinusoidal endothelium in lipoprotein metabolism, atherosclerosis, and cirrhosis. Hepatology 1995;21:863-874. 


\section{Figure legends}

Figure 1: (a) Macroscopic photographs of livers from control and choline-deficient, L-amino acid-defined (CDAA) 8-week mice. (b) Body weight, liver/body weight ratio, spleen/body weight ratio, and visceral (epididymal) fat/body weight ratio in control (white circles) and CDAA-fed mice (black boxes) during the experimental feeding period of 8 weeks. Data are presented as means \pm SEM. ${ }^{*} P<0.05$ compared with each corresponding time point of the control mice. ${ }^{* *} P<0.01$ compared with the control mice. (c) Serum levels of alanine aminotransferase (ALT), fasting blood glucose, alkaline phosphatase (ALP), total bilirubin, triglyceride (TG), total cholesterol, and free fatty acid (FFA) in control mice ( 0 weeks; white bar) and CDAA-fed mice (black bar) after 1 , 4, and 8 weeks. ${ }^{*} P<0.05$ compared with the control $\left(0\right.$-week) mice; ${ }^{* *} P<0.01$ compared with the control ( 0 -week) mice; NS, not significant. $\mathrm{n} \geq 6$ for all groups.

Figure 2: (a) Histopathological images of livers from control and choline-deficient, L-amino acid-defined (CDAA)-fed mice after 1, 4, and 8 weeks. Hematoxylin and eosin $(\mathrm{H} \& \mathrm{E})$ staining (top row; bars $=100 \mu \mathrm{m})$, Oil red O staining (second row; bars $=25$ $\mu \mathrm{m}$ ), Azan staining (third row; bars $=50 \mu \mathrm{m}$ ), and reticulin staining (bottom row; bars $=$ $25 \mu \mathrm{m})$ are shown. Arrows indicate inflammatory cell infiltration (steatohepatitis). Arrowheads indicate reticulin-positive areas (type III collagen) in the intralobular 
lesions. pv, portal vein; cv, central vein. (b) Nonalcoholic fatty liver disease activity scores (steatosis, inflammation, and ballooning) and fibrosis stage in control and CDAA-fed mice after 1,4 , and 8 weeks. Data are presented as means \pm SEM. ${ }^{* *} P<0.01$ compared with the control mice. $\mathrm{n} \geq 5$ for all groups. N.D, not detected.

Figure 3: (a) Scanning electron microscopic images (top row, low-magnification images, bars $=5 \mu \mathrm{m}$; bottom row, high-magnification images of boxed areas, bars $=1$ $\mu \mathrm{m})$ of liver sinusoidal endothelial cells in control and choline-deficient, L-amino acid-defined (CDAA)-fed mice after 1, 4, and 8 weeks. Arrows indicate fenestrae grouped into sieve plates. Arrowheads indicate gaps. (b) Porosity in control (white bar) and CDAA-fed mice (black bar) after 1,4 , and 8 weeks. ${ }^{*} P<0.05$ compared with the control ( 0 -week) mice; ${ }^{* *} P<0.01$ compared with the control. $\mathrm{n} \geq 3$ for all groups. Fifteen random fields $(\times 10000$ magnification) were analyzed for each group. (c) Immunohistochemical staining for cluster of differentiation (CD)31 (top row) and CD34 (bottom row) in control and CDAA-fed mice after 1, 4, and 8 weeks. Asterisks indicate the internal positive control-stained areas (Glisson's capsules). Arrowheads indicate the positive areas for each antibody. Bars $=50 \mu \mathrm{m}$. (d) Quantitative analyses for each antibody-positive area in the control (white bar) and CDAA-fed mice (black bar) after 1, 
4, and 8 weeks. Data are presented as means \pm SEM. ${ }^{*} P<0.05$ compared with the control $\left(0\right.$-week) mice; ${ }^{* *} P<0.01$ compared with the controls. Ten random fields were analyzed for each group ( $\times 200$ magnification).

Figure 4: (a) Toluidine blue staining of resin-embedded liver sections for control and choline-deficient, L-amino acid-defined (CDAA)-fed mice after 4 weeks. Grey staining indicates lipid droplets. Arrowheads indicate hepatic stellate cells (lipid storing cells; Ito cells). The arrow indicates lipogranuloma. Bar $=25 \mu \mathrm{m}$. (b) Transmission electron microscopic images of livers from control and CDAA-fed mice after 4 weeks. Black arrowheads (top right panel) indicate elongated pseudopods of Kupffer cell with lipid phagocytosis. White arrowheads (bottom right panel) highlight collagen release. Bars = $5 \mu \mathrm{m} . \mathrm{H}$, hepatocyte; HSC, hepatic stellate cell; K, Kupffer cell; LSEC, liver sinusoidal endothelial cell. (c) Immunohistochemical staining for F4/80 (top row) and $\alpha$-smooth muscle actin ( $\alpha$ SMA; bottom row) in control and CDAA-fed mice after 1,4 , and 8 weeks. Arrowheads indicate positive staining of each antibody. Asterisks indicate the internal positive control-stained areas (smooth muscle of arteries). The right panel shows the quantitative analyses of each antibody-positive stained area in control (white bar) and CDAA-fed mice (black bar) after 1, 4, and 8 weeks. Data are presented as 
means \pm SEM. ${ }^{* *} P<0.01$ compared with the control $\left(0\right.$-week) mice; ${ }^{* * *} P<0.001$ compared with the controls. Ten random fields were analyzed for each group $(\times 200$ magnification). (d) Quantitative RT-PCR analysis in control and CDAA-fed mice after 1 and 4 weeks. Values are normalized to GAPDH expression and are presented as means \pm SEM. ${ }^{*} P<0.05$ compared with the control $\left(0\right.$-week) mice; ${ }^{* *} P<0.01$ compared with the control; ${ }^{* * *} P<0.001$ compared with the control.

Figure 5: (a) Macroscopic photographs of livers from control 22-week and choline-deficient, L-amino acid-defined (CDAA) 22-week mice. The CDAA-fed mice showed atrophic livers with rough surfaces and pale yellow discoloring, indicating cirrhosis. (b) Scanning electron microscopic images of liver sinusoidal endothelial cells in control 0-week, control 22-week, and CDAA 22-week mice. Arrowheads indicate fenestrae grouped into sieve plates. Asterisks indicate collagen deposits in the space of Disse. Bars $=1 \mu \mathrm{m}$. (c) Porosity in control 0-week, control 22-week, and CDAA 22-week mice. Data are presented as means \pm SEM. ${ }^{*} P<0.05$ compared with the control 0-week mice; ${ }^{\#} P<0.01$ compared with the control 22 -week mice. $\mathrm{n} \geq 3$ for all groups. Fifteen random fields were analyzed for each group (×10 000 magnification). 
Figure 6: (a) Histopathological images of livers from control 8-week, choline-deficient, L-amino acid-defined (CDAA) 8-week, and recovery 4-week mice (8 weeks of CDAA feeding followed by an additional 4 weeks of standard diet). Hematoxylin and eosin $(\mathrm{H} \& \mathrm{E})$ staining (top row; bars $=100 \mu \mathrm{m})$, Azan staining (middle row; bars $=50 \mu \mathrm{m}$ ), and reticulin staining (bottom row; bars $=25 \mu \mathrm{m}$ ) are shown. Arrows indicate inflammatory cell infiltration (steatohepatitis). Arrowheads indicate reticulin-positive areas (type III collagen) in the intralobular lesions. Asterisks indicate dead hepatocytes with crystallized lipid droplets. pv, portal vein; cv, central vein. (b) Nonalcoholic fatty liver disease activity scores (steatosis, inflammation, and ballooning) and fibrosis stage in control 8-week, CDAA 8-week, and recovery 4-week mice. Data are presented as means \pm SEM. ${ }^{* *} P<0.01$ compared with the control mice; ${ }^{\#} P<0.05$ compared with the CDAA 8-week mice; ${ }^{\# \# ~} P<0.01$ compared with the CDAA 8-week mice; $\mathrm{n} \geq 5$ for all groups. N.D, not detected. (c) Scanning electron microscopic images of liver sinusoidal endothelial cells in control 8-week, CDAA 8-week, and recovery 4-week mice. Arrowheads indicate fenestrae grouped into sieve plates. Bars $=1 \mu \mathrm{m}$. (d) Porosity in control 8-week, CDAA 8-week, and recovery 4-week mice. Data are presented as means \pm SEM. ${ }^{*} P<0.05$ compared with the control mice; ${ }^{\#} P<0.05$ compared with the CDAA 8-week mice. $\mathrm{n} \geq 3$ for all groups. Fifteen random fields were analyzed for each group 
(×10 000 magnification).

Figure 7: (a) Macroscopic photographs of livers from control 8-week, high fat diet (HFD) 8-week, and HFD 22-week mice. (b) Histopathological images of livers from control, HFD 8-week, and HFD 22-week mice. Hematoxylin and eosin (H\&E) staining (top row; bars $=100 \mu \mathrm{m}$ ), Azan staining (middle row; bars $=50 \mu \mathrm{m}$ ), and reticulin staining (bottom row; bars $=25 \mu \mathrm{m}$ ) are shown. The arrow indicates inflammatory cell infiltration (steatohepatitis). Arrowheads indicate reticulin-positive areas (type III collagen) in the intralobular regions. pv, portal vein; cv, central vein. (c) Nonalcoholic fatty liver disease activity scores (steatosis, inflammation, and ballooning) and fibrosis stage in control 8-week, choline-deficient, L-amino acid-defined (CDAA) 8-week, and HFD 8-week mice. Data are presented as means \pm SEM. ${ }^{* *} P<0.01$ compared with the control mice. $n \geq 5$ for all groups. N.D, not detected. (d) Nonalcoholic fatty liver disease activity score and fibrosis stage in control 22-week, CDAA 22-week, and HFD 22-week mice. Data are presented as means \pm SEM. ${ }^{*} P<0.05$ compared with the control mice. ${ }^{* *} P<0.01$ compared with the control mice. $\mathrm{n} \geq 5$ for all groups. N.D, not detected. (e) Scanning electron microscopic images of liver sinusoidal endothelial cells in HFD 8-week, HFD 22-week, CDAA 8-week, and CDAA 22-week mice. Arrowheads indicate 
fenestrae grouped into sieve plates. Asterisks indicate collagen deposits in the space of

Disse. Bars $=1 \mu \mathrm{m}$. (f) Porosity in control, HFD, and CDAA mice after 8 and 22 weeks.

Data are presented as means \pm SEM. ${ }^{*} P<0.05$ compared with the control 22-week mice;

${ }^{\#} P<0.05$ compared with the HFD 22-week mice; ${ }^{\#} P<0.01$ compared with the HFD

8-week mice. $n \geq 3$ for all groups. Fifteen random fields were analyzed for each group (×10 000 magnification). 
Table 1. Phenotypic and biochemical findings

\begin{tabular}{|c|c|c|c|c|}
\hline & Control 8 weeks & HFD 8 weeks & CDAA 8 weeks & Recovery 4 weeks \\
\hline Body weight (g) & $27.5 \pm 0.7$ & $40.4 \pm 1.3^{\star}$ & $26.9 \pm 0.5$ & $30.3 \pm 1.4^{\#}$ \\
\hline Liver/body weight (\%) & $4.87 \pm 0.32$ & $4.34 \pm 0.20$ & $6.04 \pm 0.84^{*}$ & $4.89 \pm 0.26^{\#}$ \\
\hline Spleen/body weight (\%) & $0.30 \pm 0.01$ & $0.20 \pm 0.01^{*}$ & $0.41 \pm 0.03^{\star}$ & $0.49 \pm 0.08^{\#}$ \\
\hline Epididymal/body fat (\%) & $1.32 \pm 0.28$ & $5.75 \pm 0.26^{*}$ & $1.99 \pm 0.30^{*}$ & $2.11 \pm 0.33$ \\
\hline ALT (IU/L) & $64.7 \pm 21.5$ & $46.3 \pm 12.3$ & $162.0 \pm 19.4^{\star}$ & $26.5 \pm 5.4^{\#}$ \\
\hline $\operatorname{ALP}(I U / L)$ & $234.2 \pm 13.6$ & $142.7 \pm 11.7^{\star}$ & $364.3 \pm 14.0^{\star}$ & $213.7 \pm 14.6^{\#}$ \\
\hline Cholesterol (mg/dl) & $57.6 \pm 4.6$ & $125.1 \pm 8.4^{*}$ & $74.8 \pm 4.8^{*}$ & $69.7 \pm 8.2$ \\
\hline Triglycerides (mg/dl) & $68.2 \pm 7.3$ & $44.1 \pm 7.7^{\star}$ & $37.2 \pm 1.2^{*}$ & $36.0 \pm 5.5$ \\
\hline Free fatty acids (mg/dl) & $142.2 \pm 28.5$ & $328.7 \pm 30.9^{*}$ & $137.2 \pm 12.1$ & $278.0 \pm 64.1^{\#}$ \\
\hline Bilirubin (mg/dl) & $0.02 \pm 0.01$ & $0.05 \pm 0.004^{*}$ & $0.11 \pm 0.01^{*}$ & $0.04 \pm 0.02^{\#}$ \\
\hline \multirow[t]{2}{*}{ Glucose (mg/dl) } & $165.4 \pm 7.1$ & $251.3 \pm 29.1^{*}$ & $203.0 \pm 6.1^{*}$ & $162.0 \pm 14.1^{\#}$ \\
\hline & Control 22 weeks & HFD 22 weeks & CDAA 22 weeks & \\
\hline Body weight (g) & $35.1 \pm 2.1$ & $53.5 \pm 1.7^{\star}$ & $28.6 \pm 1.4^{*}$ & \\
\hline Liver/body weight (\%) & $4.04 \pm 0.15$ & $5.96 \pm 0.24^{*}$ & $7.24 \pm 0.39^{*}$ & \\
\hline Spleen/body weight (\%) & $0.29 \pm 0.02$ & $0.24 \pm 0.01^{*}$ & $0.48 \pm 0.03^{*}$ & \\
\hline Epididymal/body fat (\%) & $3.87 \pm 0.46$ & $5.10 \pm 0.38^{*}$ & $1.92 \pm 0.41^{*}$ & \\
\hline $\mathrm{ALT}(\mathrm{IU} / \mathrm{L})$ & $33.8 \pm 8.3$ & $148.7 \pm 26.1^{*}$ & $45.2 \pm 15.2$ & \\
\hline ALP (IU/L) & $160.5 \pm 8.1$ & $183.0 \pm 24.0$ & $300.4 \pm 176.3$ & \\
\hline Cholesterol (mg/dl) & $84.3 \pm 6.7$ & $210.0 \pm 22.2^{*}$ & $56.8 \pm 14.9$ & \\
\hline Triglycerides (mg/dl) & $58.3 \pm 11.7$ & $28.3 \pm 3.9^{\star}$ & $22.7 \pm 4.2^{*}$ & \\
\hline Free fatty acids $(\mathrm{mg} / \mathrm{dl})$ & $380.0 \pm 102.3$ & $280.3 \pm 51.3$ & $363.5 \pm 91.0$ & \\
\hline Bilirubin (mg/dl) & $0.03 \pm 0.01$ & $0.01 \pm 0.003^{*}$ & $0.14 \pm 0.09^{*}$ & \\
\hline Glucose (mg/dl) & $187.0 \pm 31.3$ & $241.3 \pm 36.7^{\star}$ & $170.0 \pm 15.8$ & \\
\hline
\end{tabular}

Results are presented as means \pm SEM ( $n \geq 4$ for each group).

HFD: high fat diet; CDAA; choline-deficient L-amino acid-defined diet;

ALT: alanine aminotransferase; ALP: alkaline phosphatase.

${ }^{*} P<0.05$ vs. each control, ${ }^{\#} P<0.05$ vs. CDAA 8-week mice. 


\section{Figure 1}

a

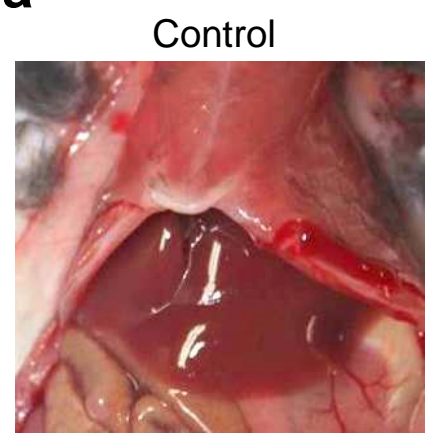

CDAA 8 w

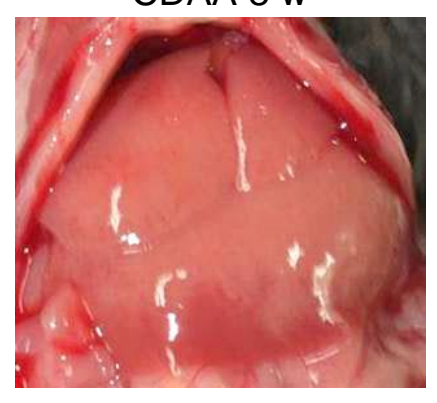

b
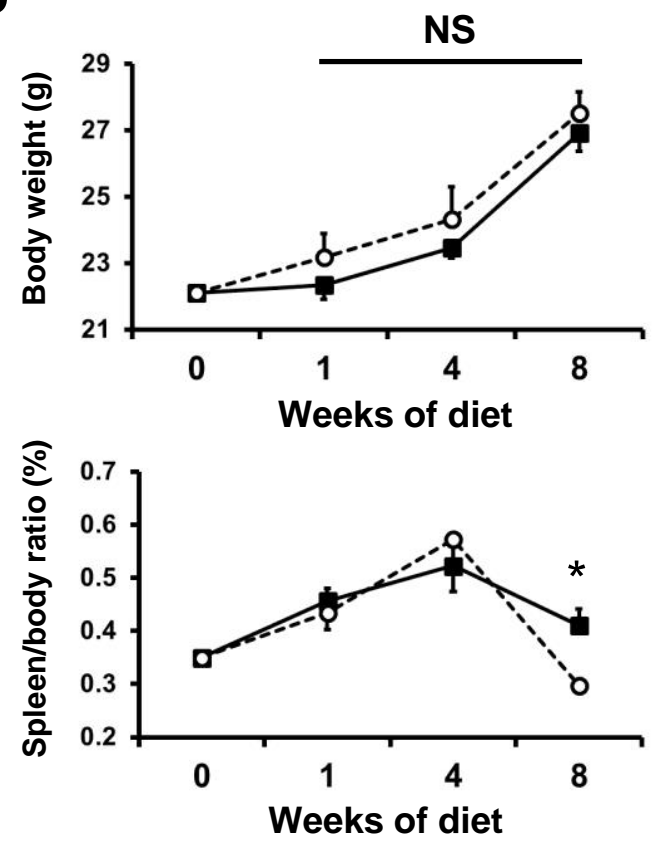

Control
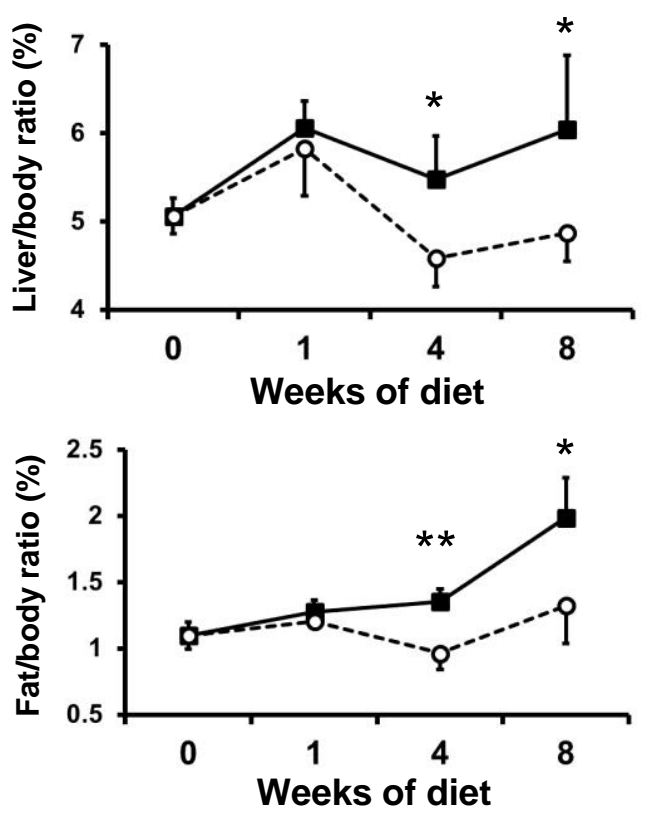

CDAA

C
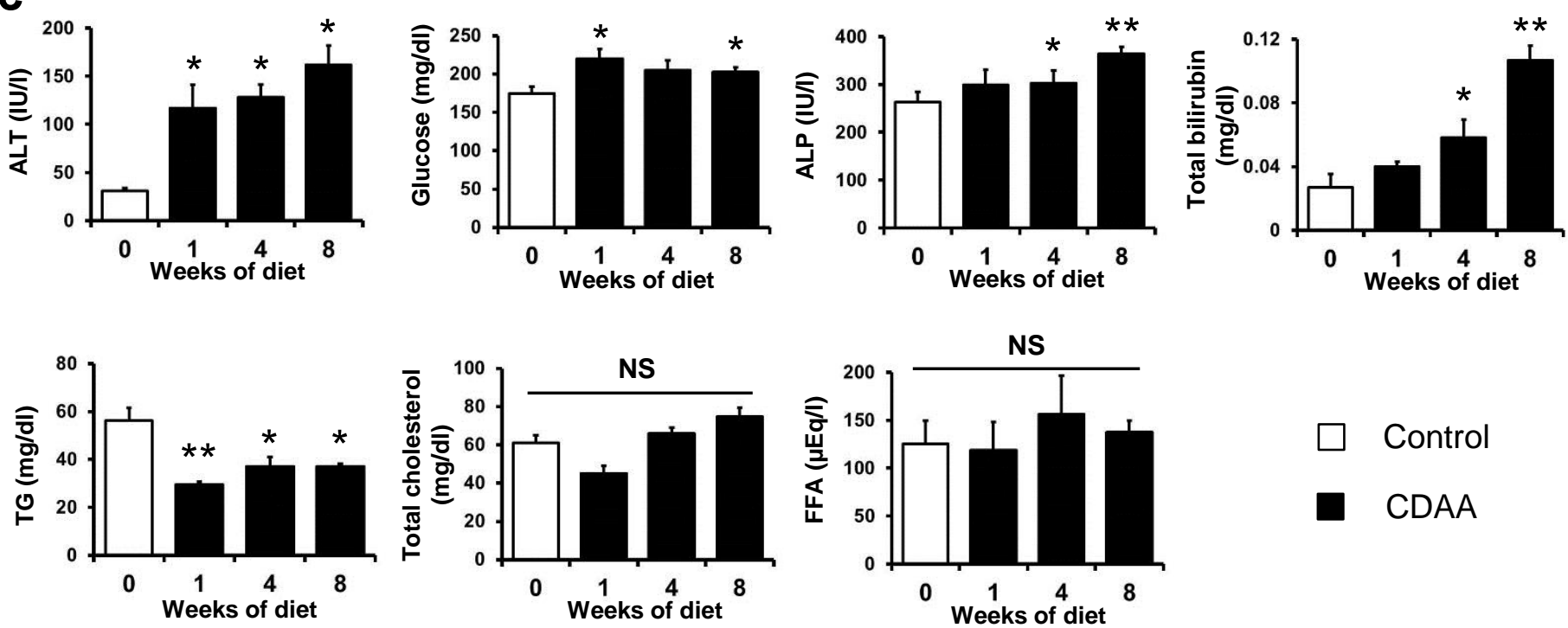

Control

- CDAA 


\section{Figure 2}

a

Control

CDAA 1 w

CDAA $4 \mathrm{w}$

CDAA 8 w
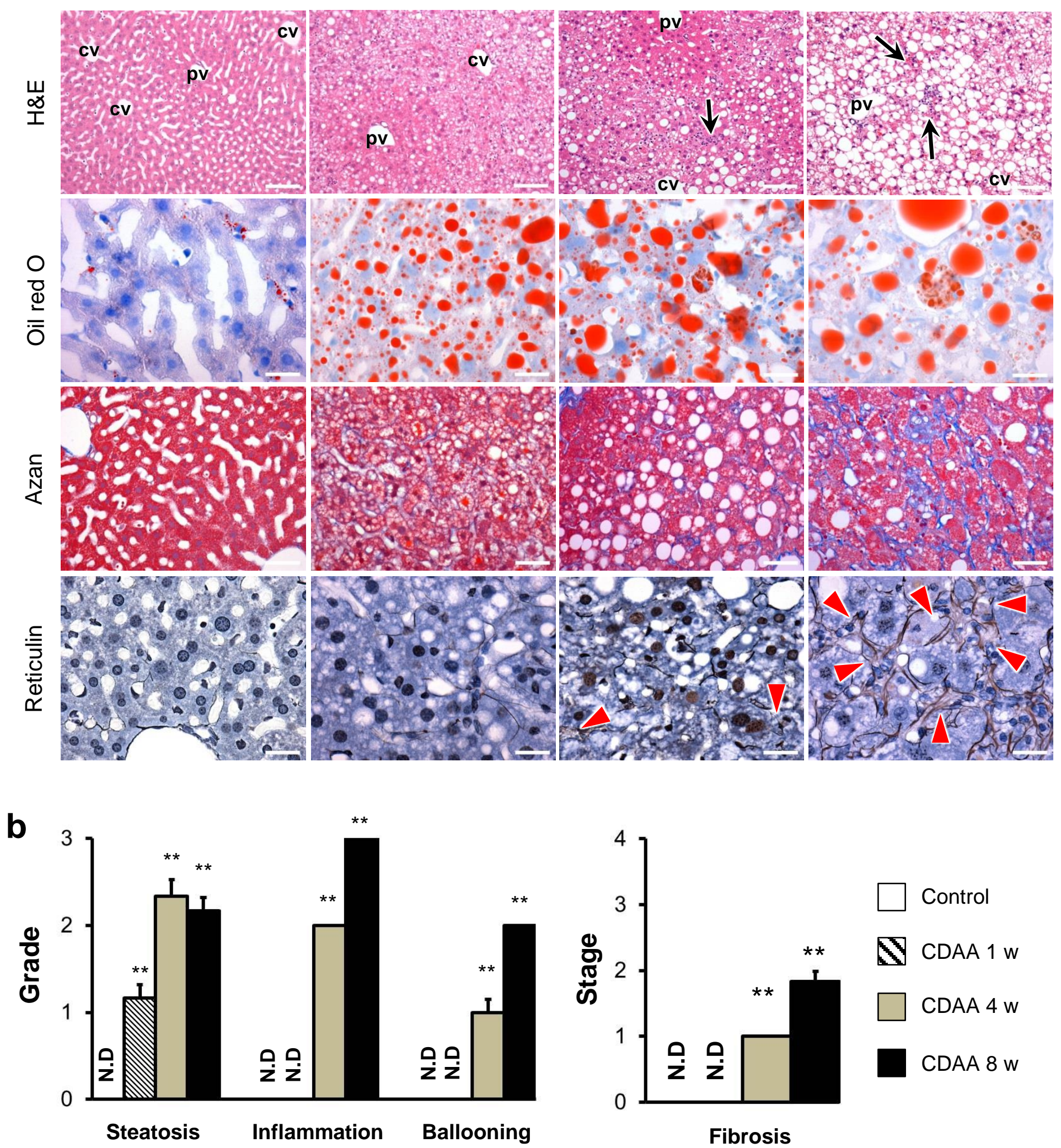

Fibrosis

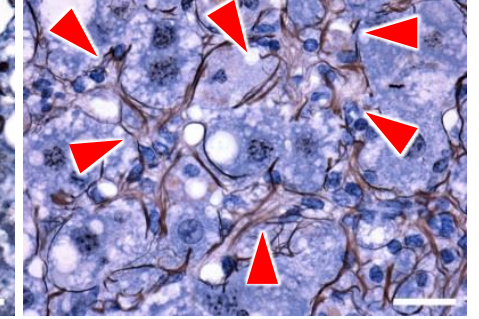

Fibrosis 
Figure 3

a

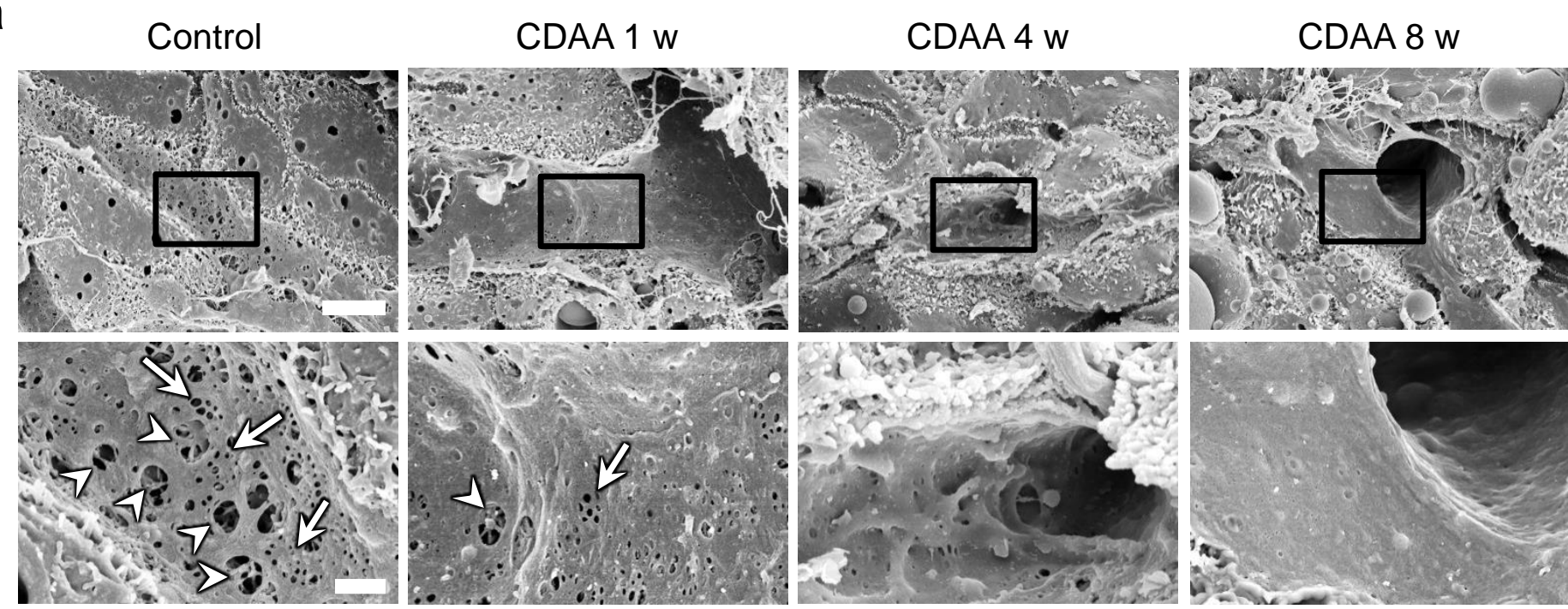

b

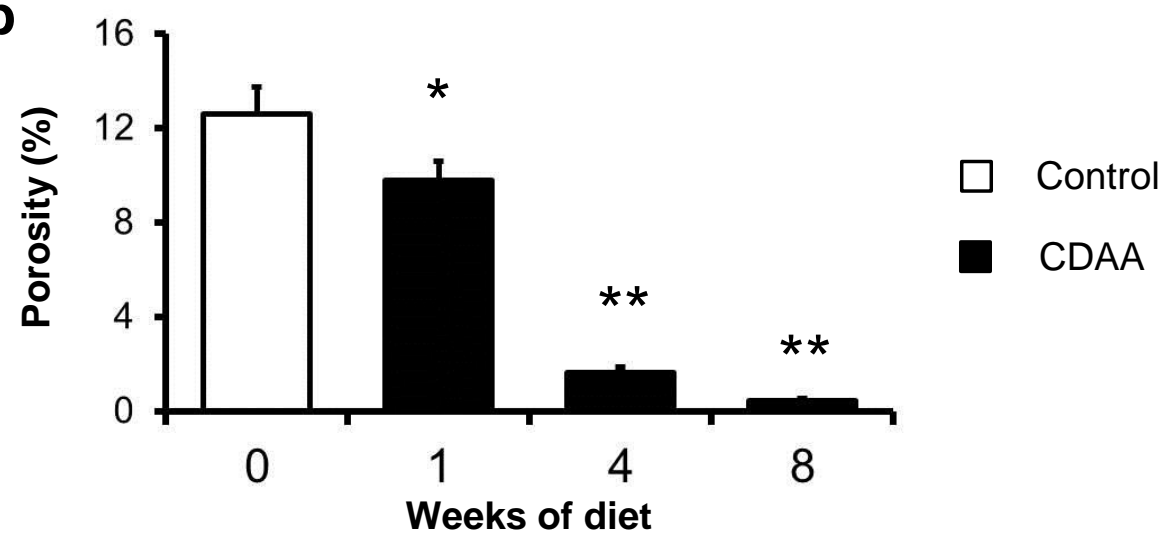

C

Control

CDAA 1 w

CDAA 4 w

CDAA 8 w

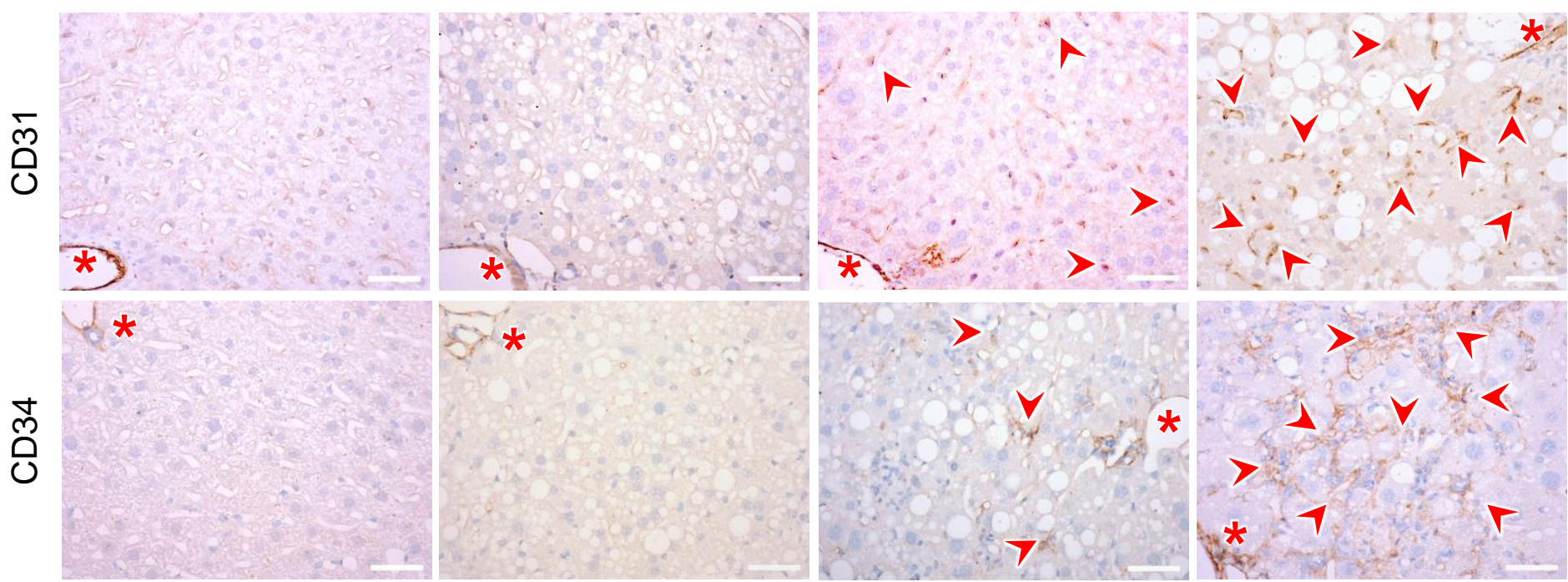

d
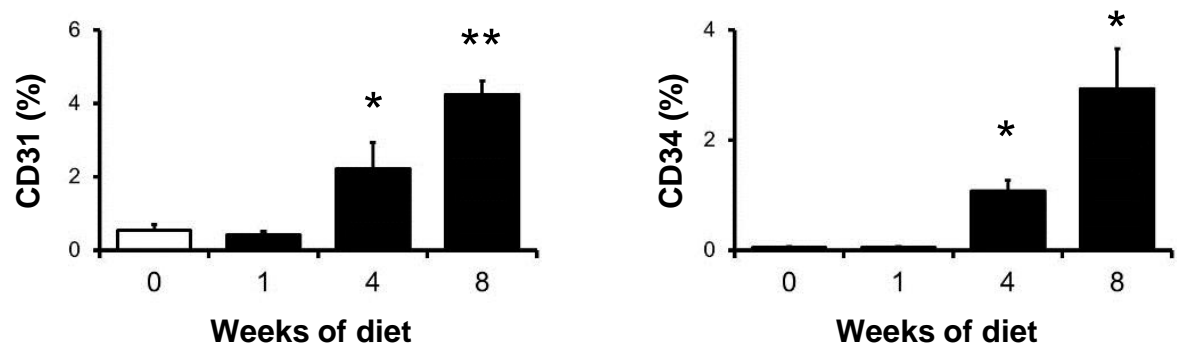

$\square$ Control

- CDAA 


\section{Figure 4}

a

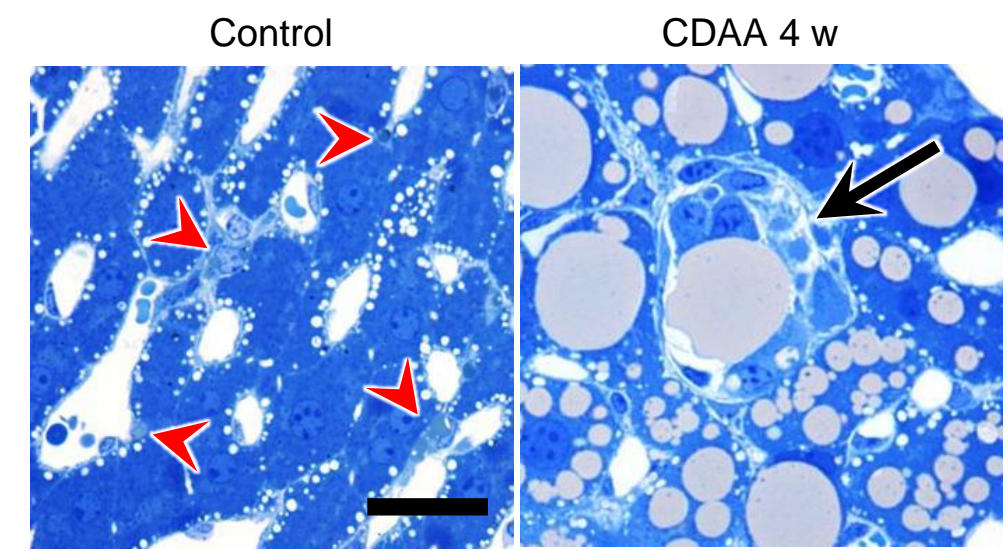

b
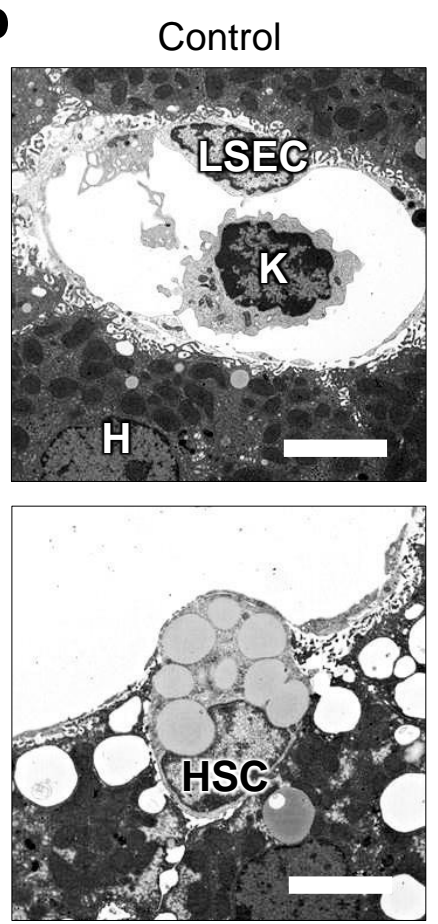
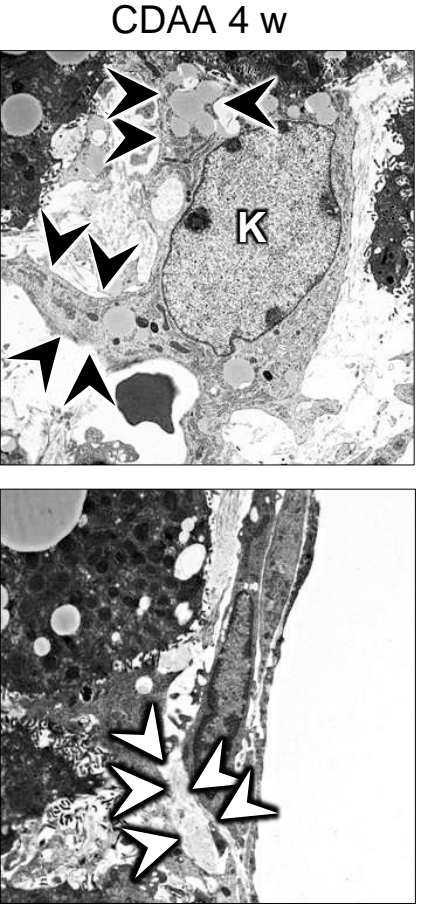

C
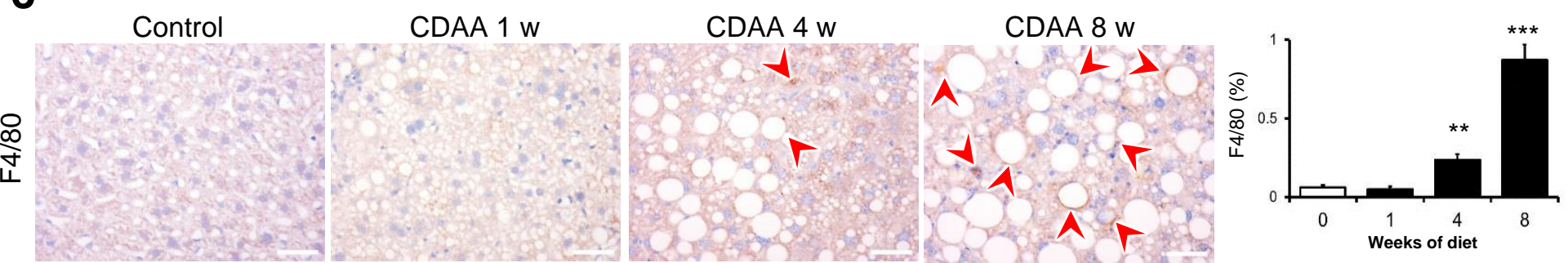

$\sum_{0}^{\mathbb{D}}$
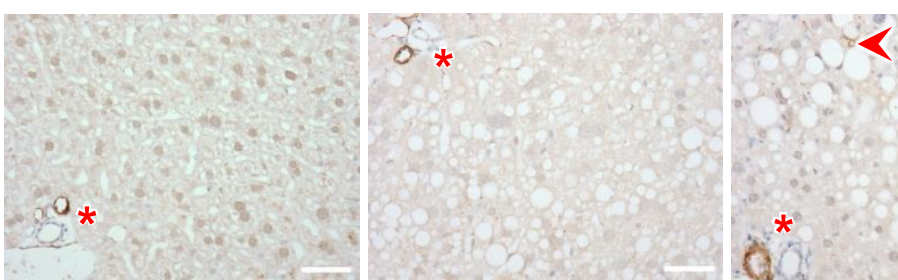

d

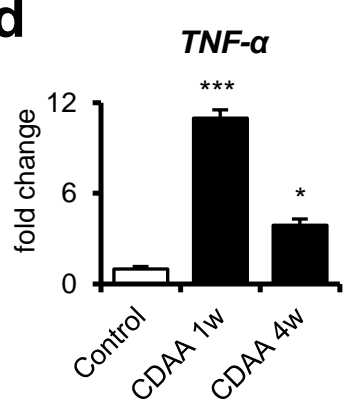

IL-6

MCP-1

Vegfr2

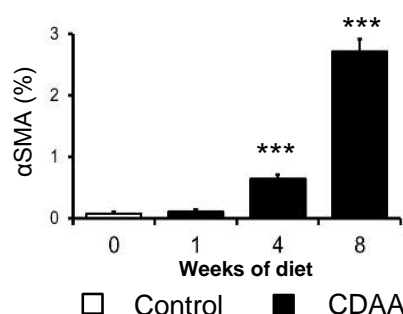

$\square$ Contro

aSMA
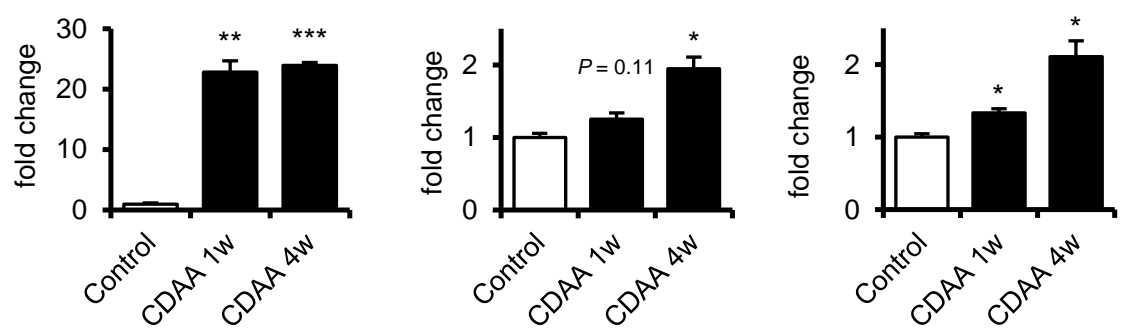

TIMP-1

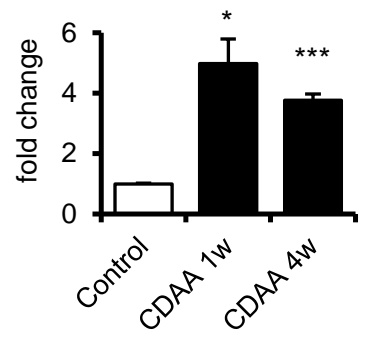

IL-1 $\beta$

Col1a1

Col4a1
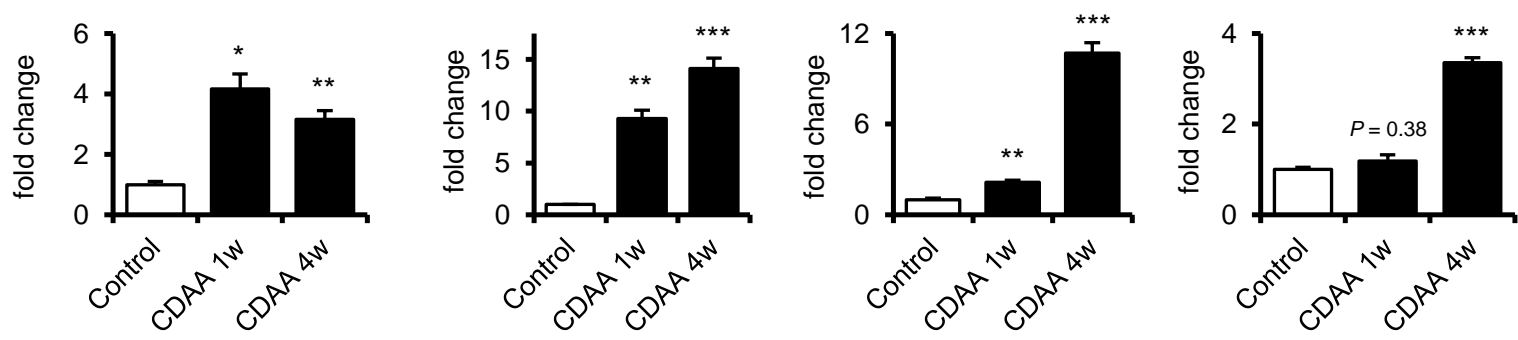
a

Control 22 w

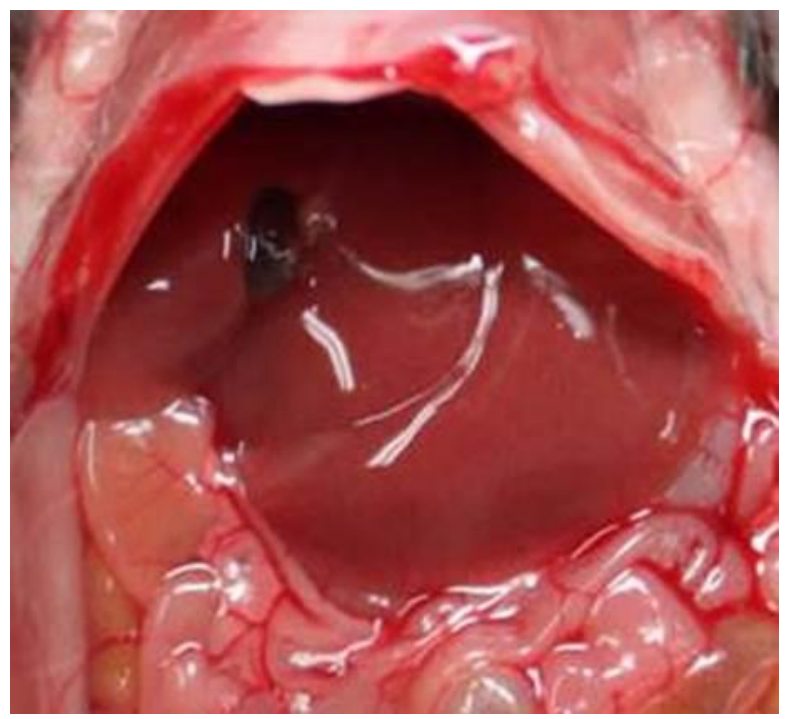

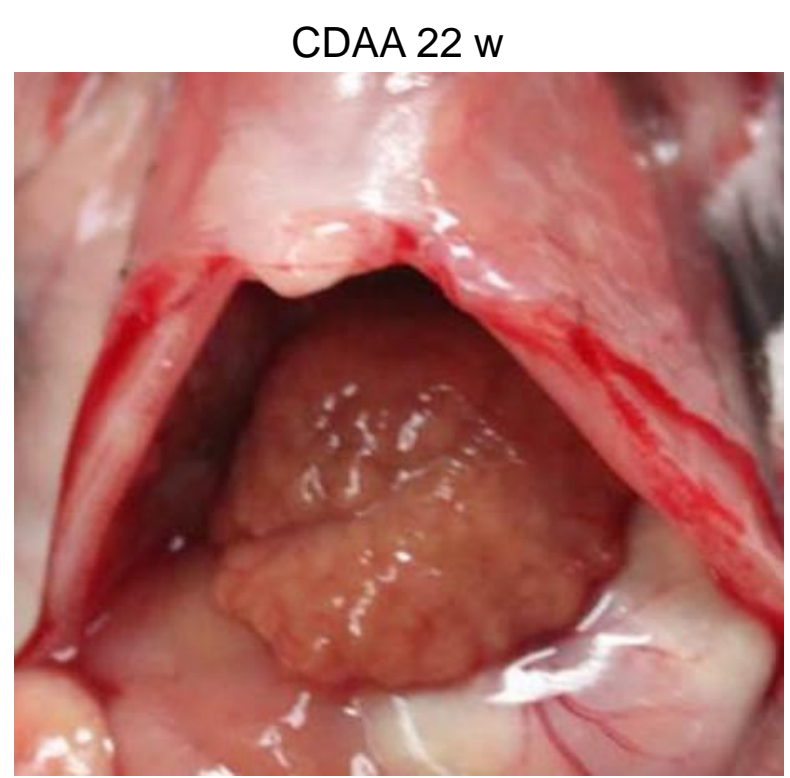

b

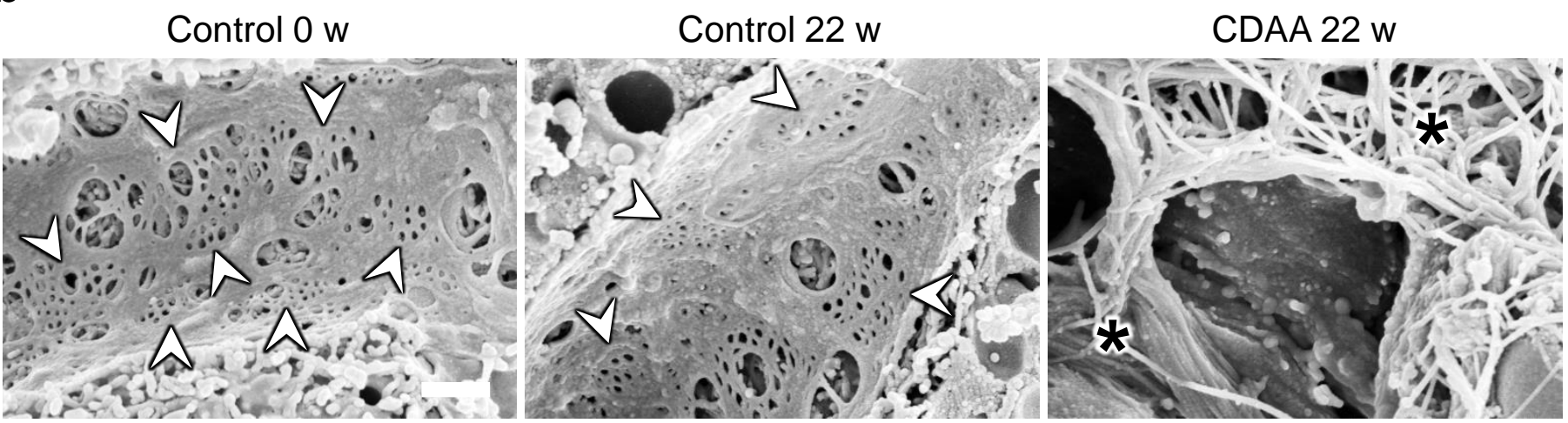

C

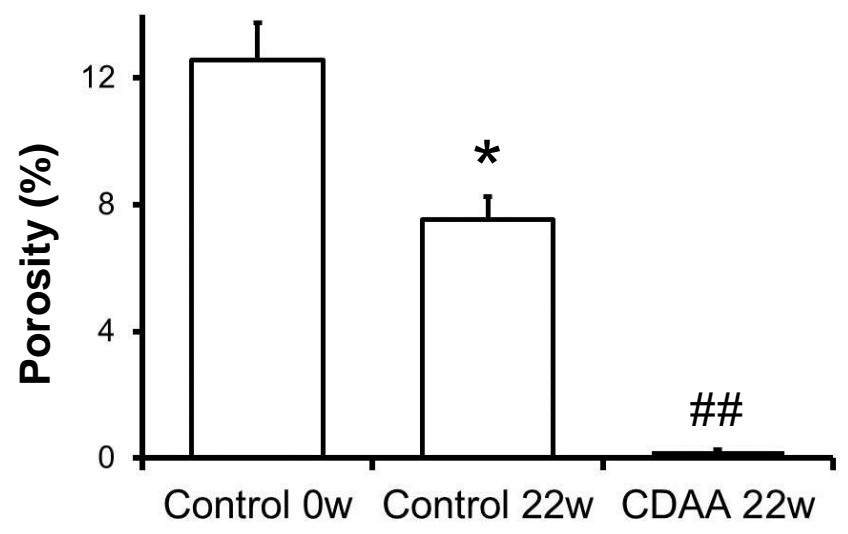




\section{Figure 6}

a

Control 8 w

CDAA 8 w

Recovery 4 w
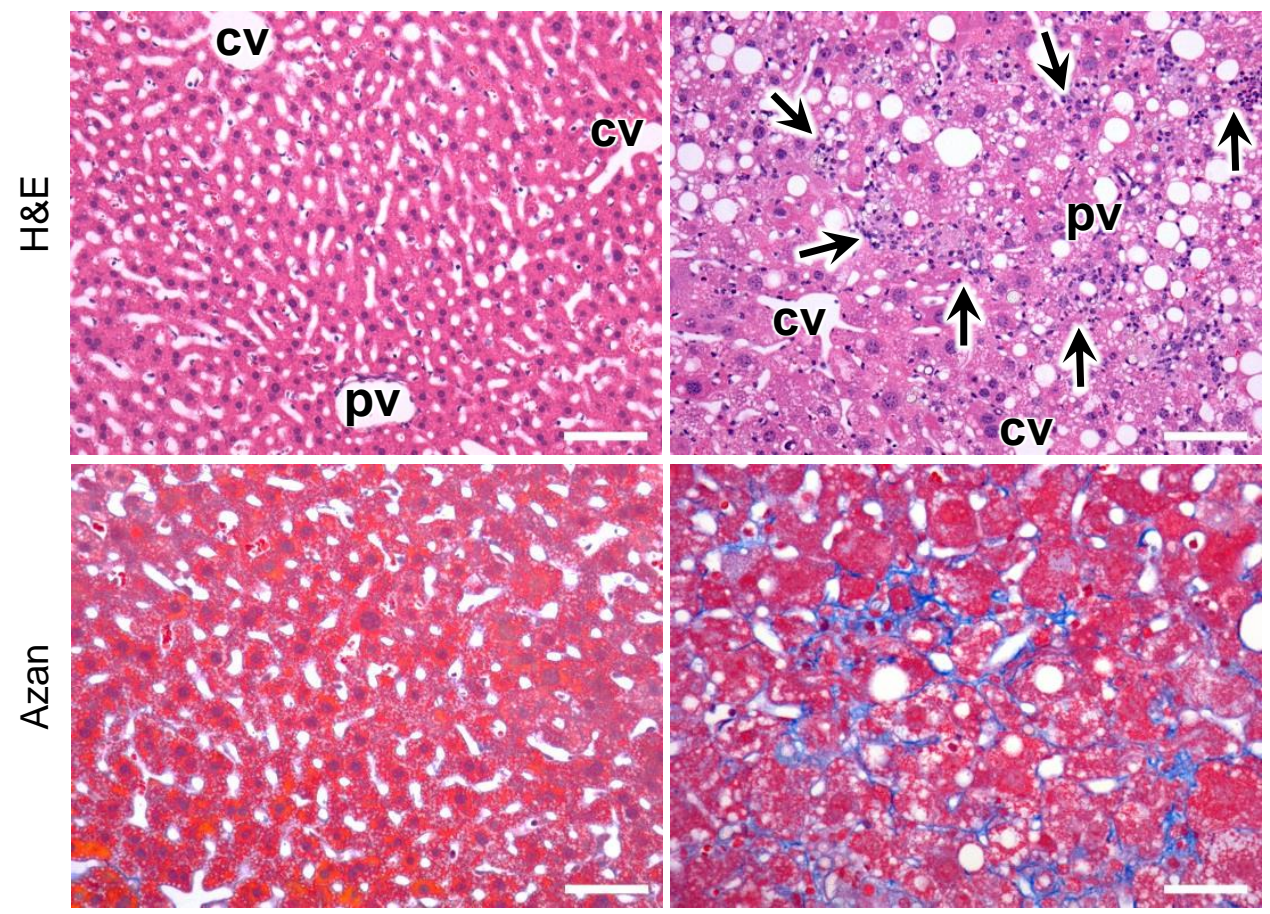

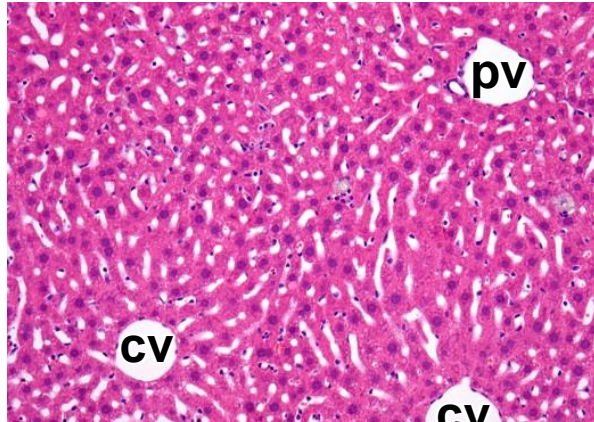
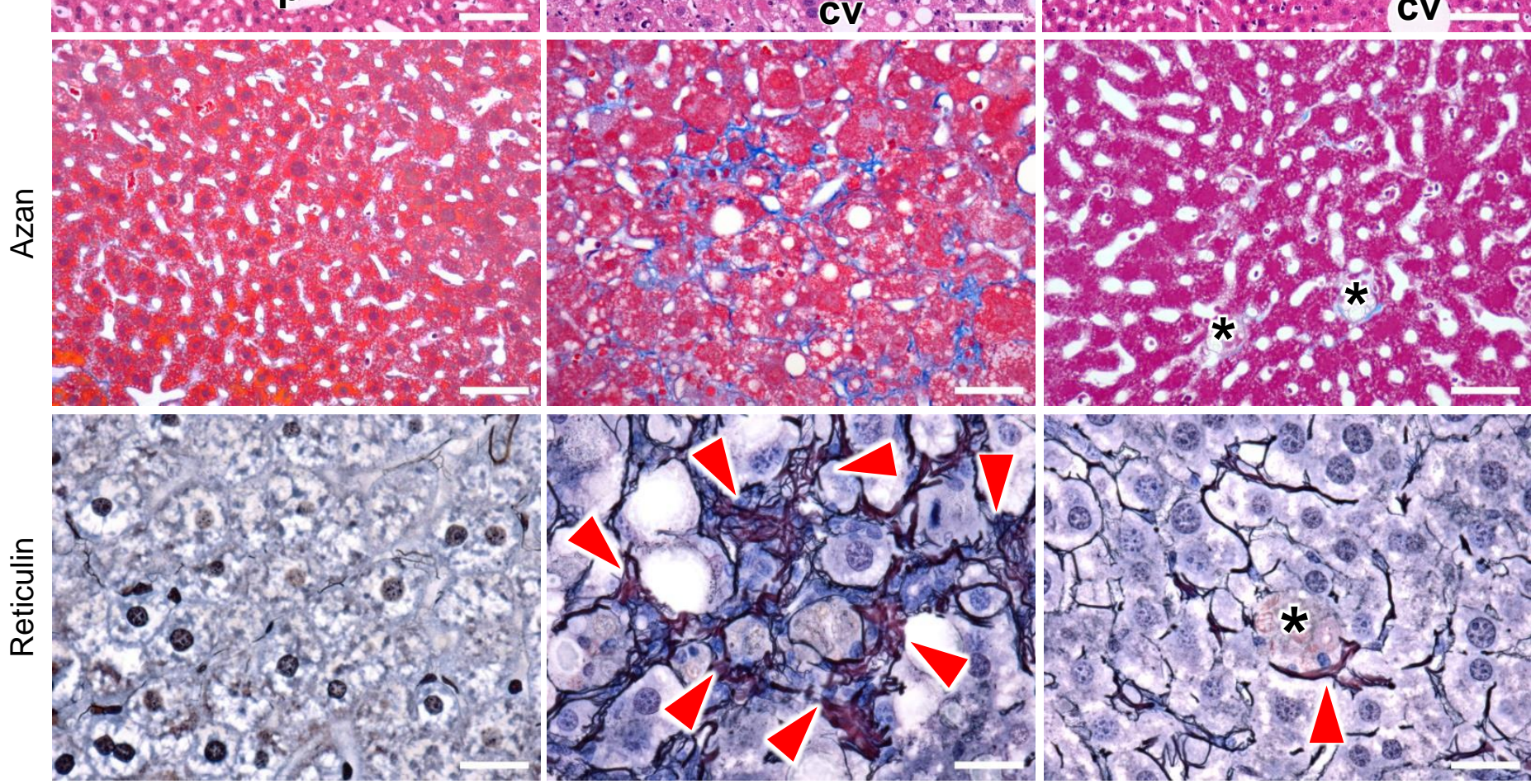

b

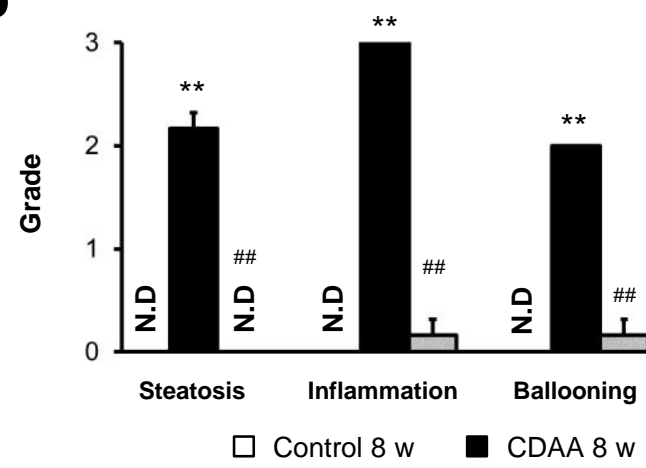

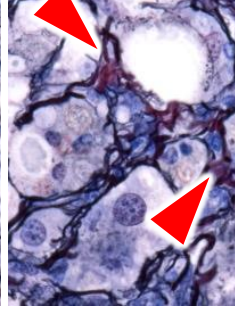

1

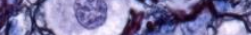

1. fol

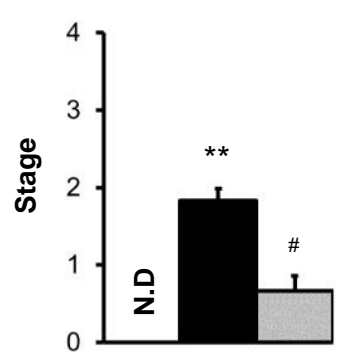

Fibrosis

$\square$ Recovery $4 \mathrm{w}$ d

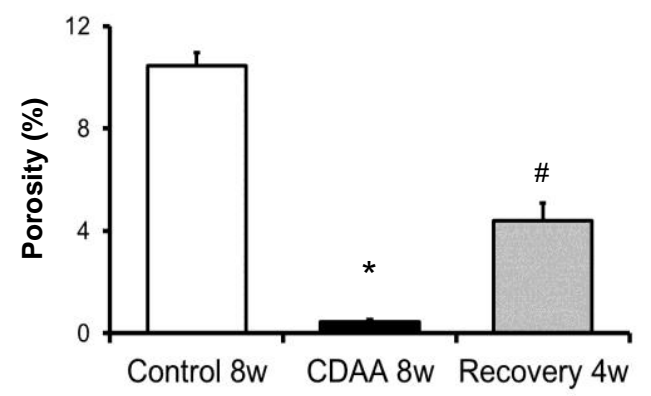

C Control 8 w

CDAA 8 w

Recovery 4 w
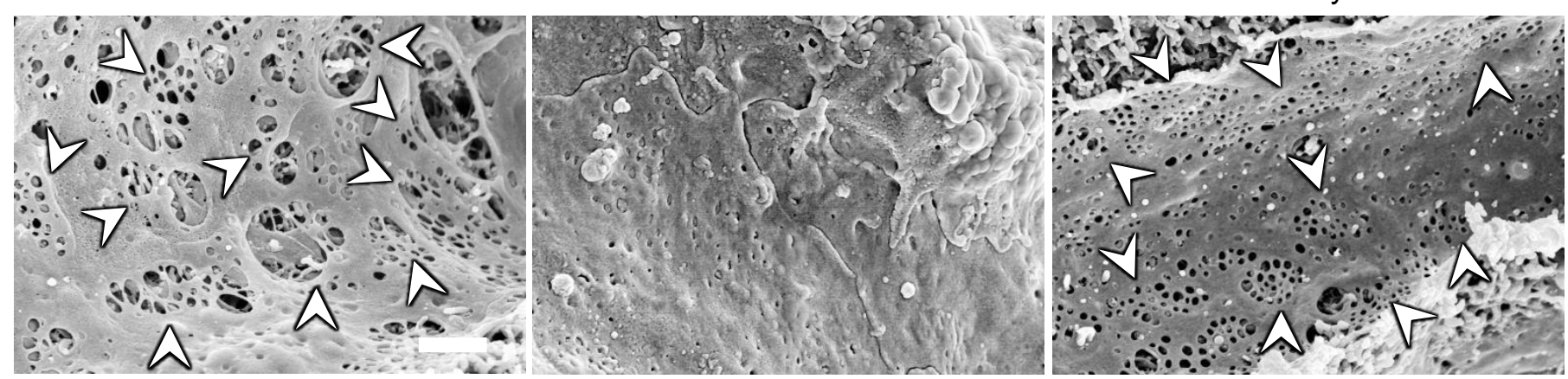


\section{Figure 7}
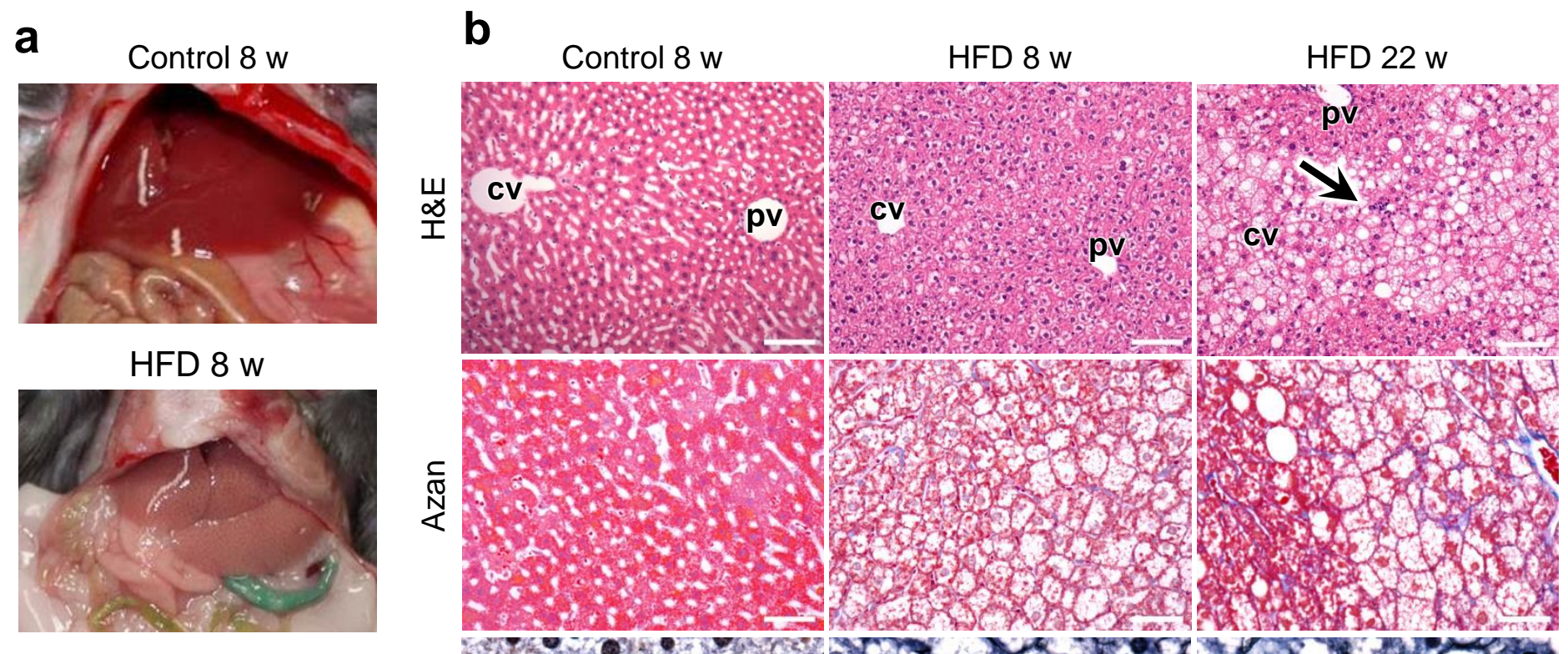

HFD 22 w
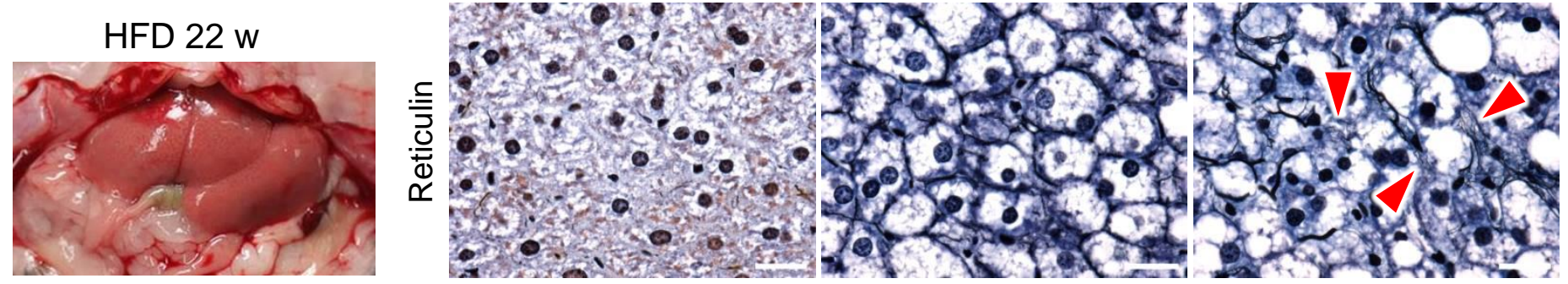

C

d
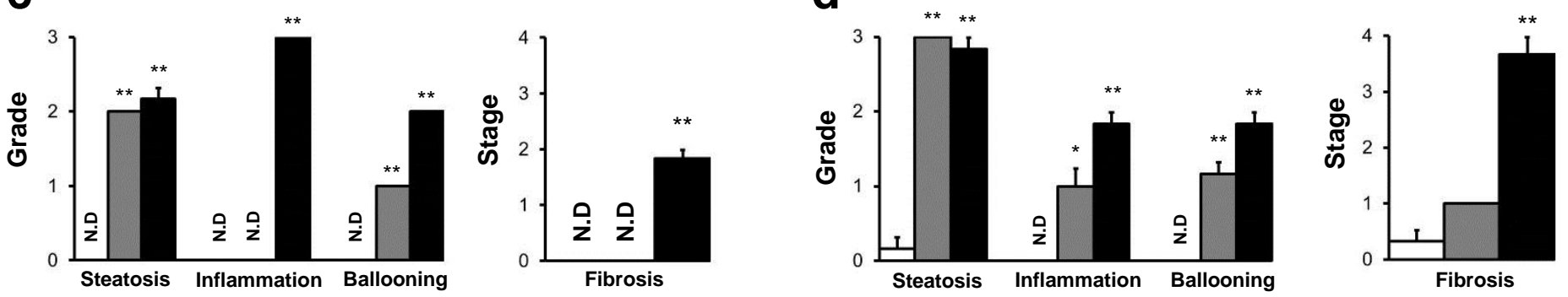

$\square$ Control 8w $\quad \square$ HFD 8 w

- CDAA 8 w

$\square$ Control $22 \mathrm{w}$

口 HFD 22 w

- CDAA $22 \mathrm{w}$

e

8 weeks
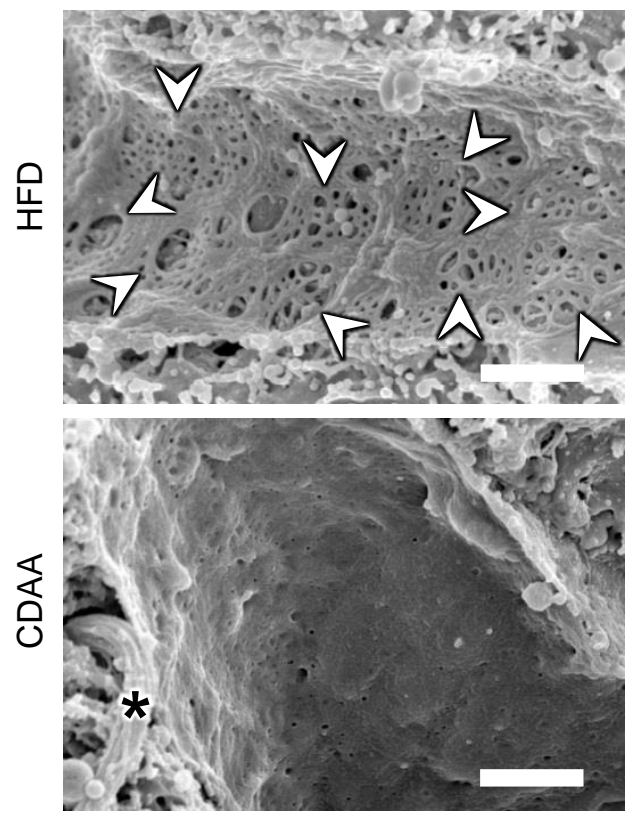

22 weeks

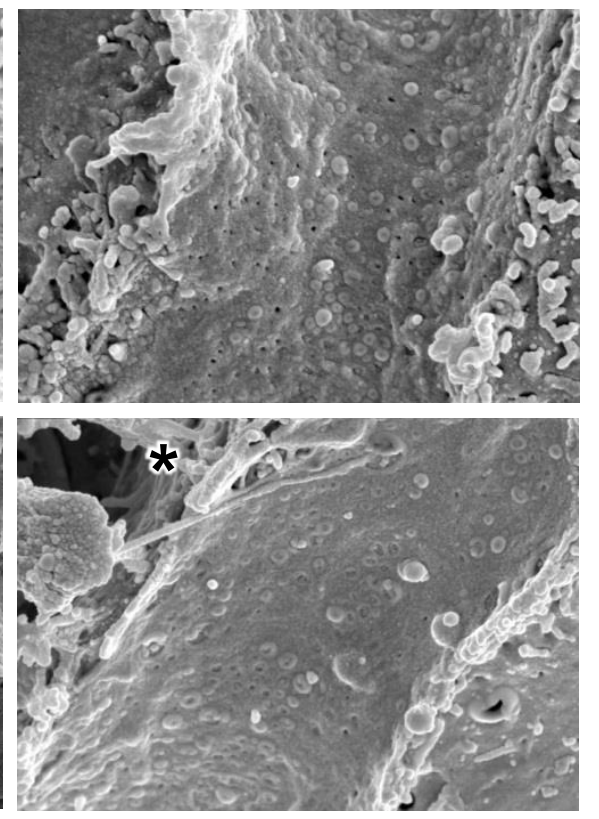

\section{f}
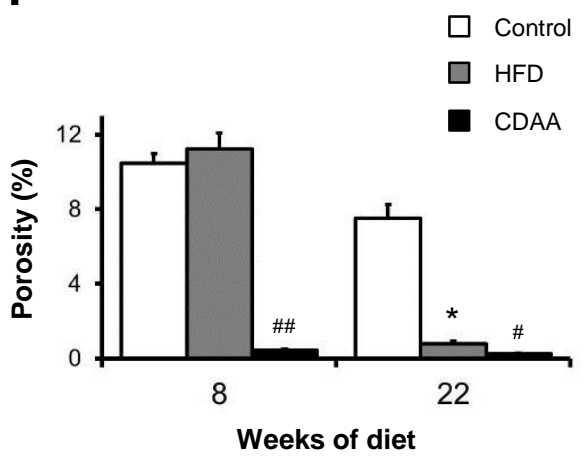


\section{Supplementary Table 1}

Supplementary Table 1. RT-PCR primers for analysis

\begin{tabular}{|c|c|c|}
\hline Gene & Direction & Sequence \\
\hline$T N F-\alpha$ & Forward & СССТСАСАСТСАGАТСАТСТTСТ \\
\hline$T N F-\alpha$ & Reverse & GCTACGACGTGGGCTACAG \\
\hline IL-6 & Forward & TAGTCCTTCCTACCCCAАTTTCC \\
\hline IL-6 & Reverse & TTGGTCCTTAGCCACTCCTTC \\
\hline$M C P-1$ & Forward & CTTCTGGGCCTGCTGTTCA \\
\hline MCP-1 & Reverse & CCAGCCTACTCATTGGGATCA \\
\hline Vegfr2 & Forward & CCTGGTAGAAGATTCAGGCATTG \\
\hline Vegfr2 & Reverse & CCTCACCCTGCGGATAGTCA \\
\hline Tek & Forward & AAGCATGCCCATCTGGTTAC \\
\hline Tek & Reverse & GCCTGCCTTCTTTCTCACAC \\
\hline$\alpha S M A$ & Forward & CCAGAGCAAGAGAGGGATCCT \\
\hline$\alpha S M A$ & Reverse & TGTCGTCCCAGTTGGTGATG \\
\hline TIMP1 & Forward & GCCCTTCGCATGGACATTTA \\
\hline TIMP1 & Reverse & CCCCGATCTGCGATGATG \\
\hline$I L-1 \beta$ & Forward & CCAGCTTCAAATCTCACAGCAG \\
\hline$I L-1 \beta$ & Reverse & CTTCTTTGGGTATTGCTTGGGATC \\
\hline Col1a1 & Forward & CACGGCTGTGTGCGATGA \\
\hline Col1a1 & Reverse & TCGCCCTCCCGTCTTTG \\
\hline Col4a1 & Forward & CCAGGATGCAACGGTACAAA \\
\hline Col4a1 & Reverse & AACGTGGCCGAGAATTTCAC \\
\hline GAPDH & Forward & AGGTCGGTGTGAACGGATTTG \\
\hline GAPDH & Reverse & TGTAGACCATGTAGTTGAGGTCA \\
\hline
\end{tabular}




\section{Supplementary Figure 1}

Control

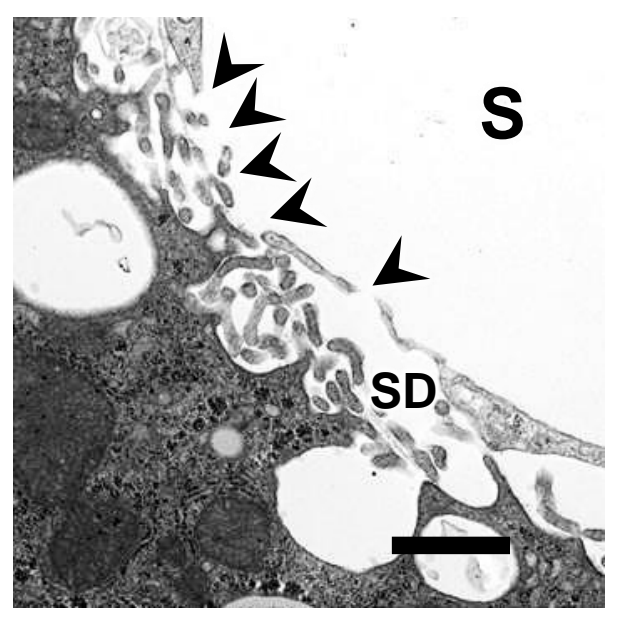

CDAA 4 w

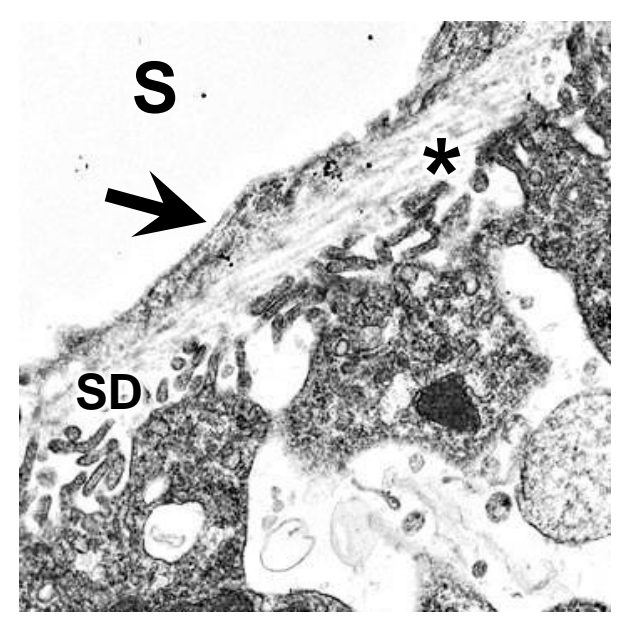

Supplementary Figure 1: Transmission electron microscopic images of liver sinusoidal endothelial cells (LSECs) in control and choline-deficient, L-amino aciddefined (CDAA) 4-week mice. Arrowheads indicate fenestrae. The arrow indicates thickened and defenestrated LSECs. The asterisk indicates collagen deposits in the space of Disse. Bar $=1 \mu \mathrm{m}$. S, sinusoid lumen; SD, space of Disse. 


\section{Supplementary Figure 2}

Control
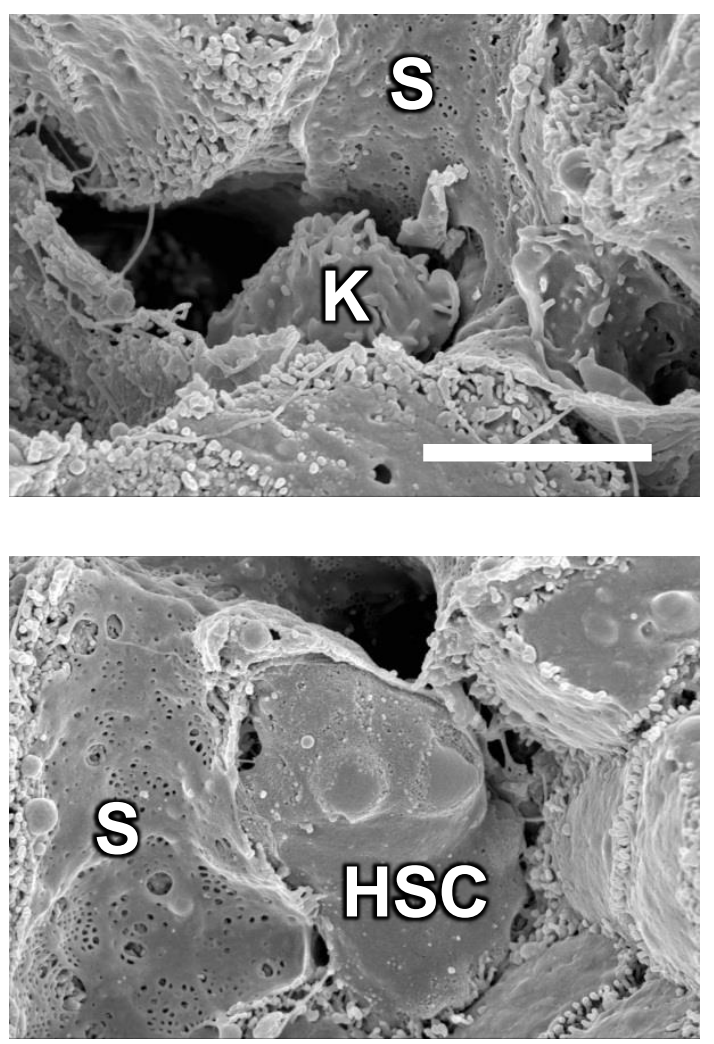

CDAA $4 \mathrm{w}$
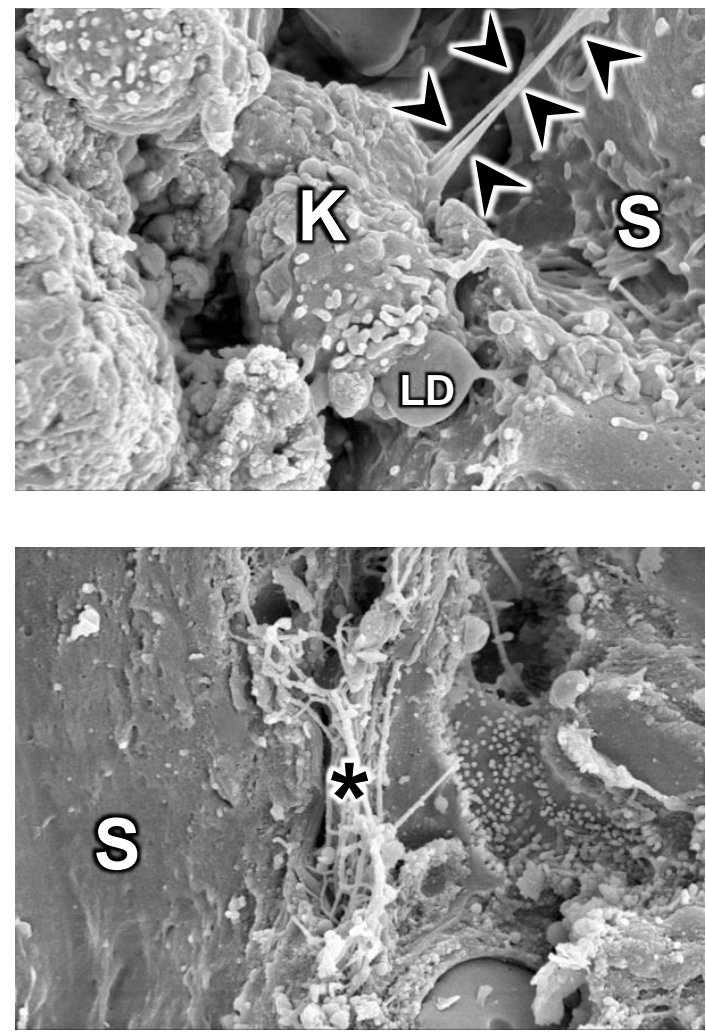

Supplementary Figure 2: Scanning electron microscopic images of Kupffer cells (top row), hepatic stellate cells (HSCs) (bottom left panel), and collagen deposits in the space of Disse (bottom right panel) in control and cholinedeficient, L-amino acid-defined (CDAA) 4-week mice. Arrowheads indicate an extended pseudopod of Kupffer cell. The uneven surface of the HSC indicates intracellular storing of lipid droplets such as vitamin A. The asterisk indicates collagen deposits in the space of Disse. Bar $=5 \mu \mathrm{m}$. HSC, hepatic stellate cell; K, Kupffer cell; LD, lipid droplet; S, sinusoid lumen. 


\section{Supplementary Figure 3}

\section{CDAA $22 \mathrm{w}$}
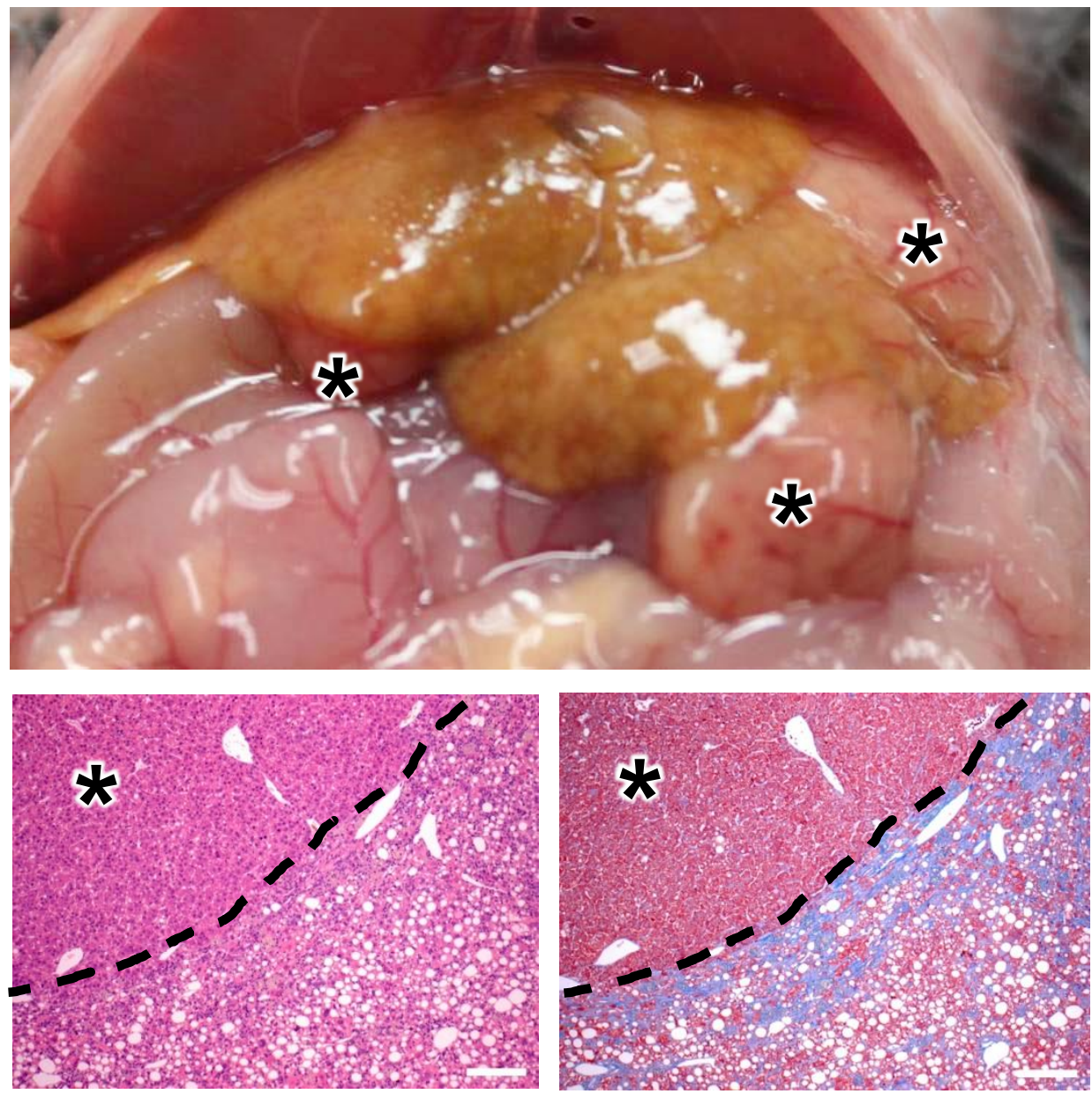

Supplementary Figure 3: Macroscopic photograph (top panel), hematoxylin and eosin staining (bottom left panel), and Azan staining (bottom right panel) of livers from choline-deficient, L-amino acid-defined (CDAA) 22-week mice. Asterisks indicate hepatocellular adenomas. The dashed lines depict the borders between the hepatocellular adenoma and non-neoplastic parenchyma. Bar $=200 \mu \mathrm{m}$. 


\section{Supplementary Figure 4}

Control $8 \mathrm{w}$

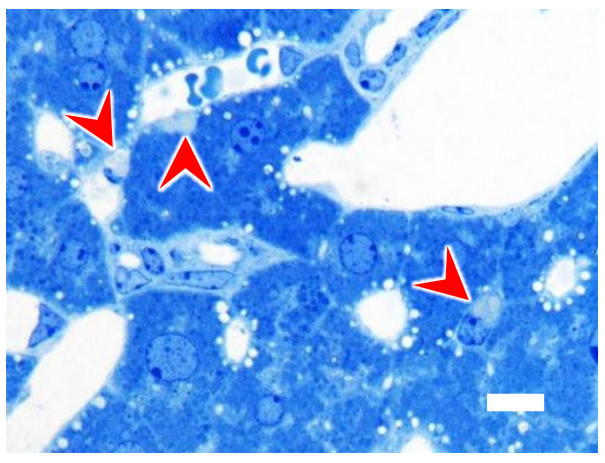

CDAA 8 w

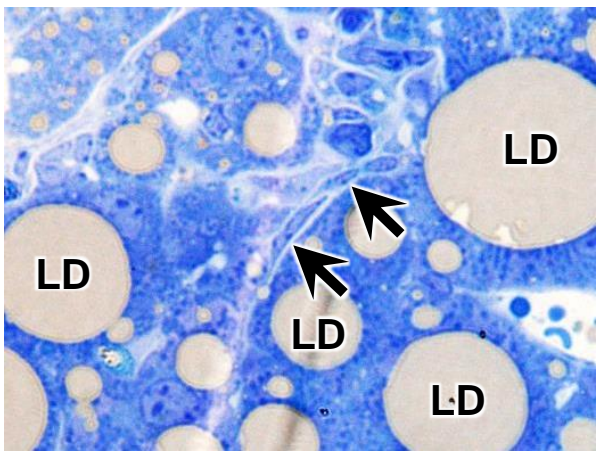

Recovery $4 \mathrm{w}$

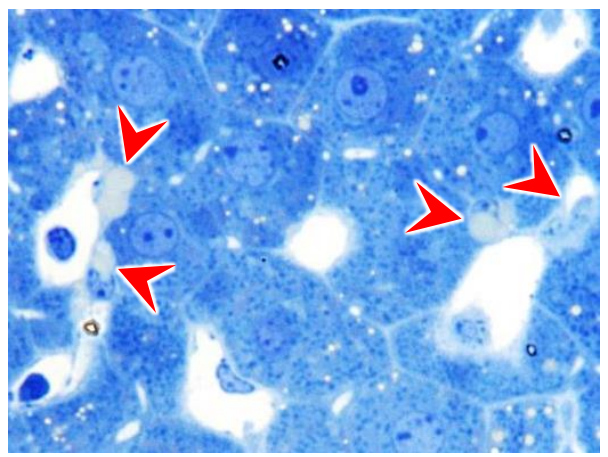

Supplementary Figure 4: Toluidine blue staining of resin-embedded liver sections for control 8-week, choline-deficient, L-amino acid-defined (CDAA) 8-week, and recovery 4-week ( 8 weeks of CDAA feeding followed by an additional 4 weeks of standard diet feeding) mice. Arrowheads indicate quiescent hepatic stellate cells (lipid storing cells) in the space of Disse. Arrows indicate activated hepatic stellate cells (myofibroblasts, spindle-shaped collagen producing cells). Bar $=10 \mu \mathrm{m}$. LD, lipid droplet. 


\section{Supplementary Figure 5}

\section{HFD 8 w}

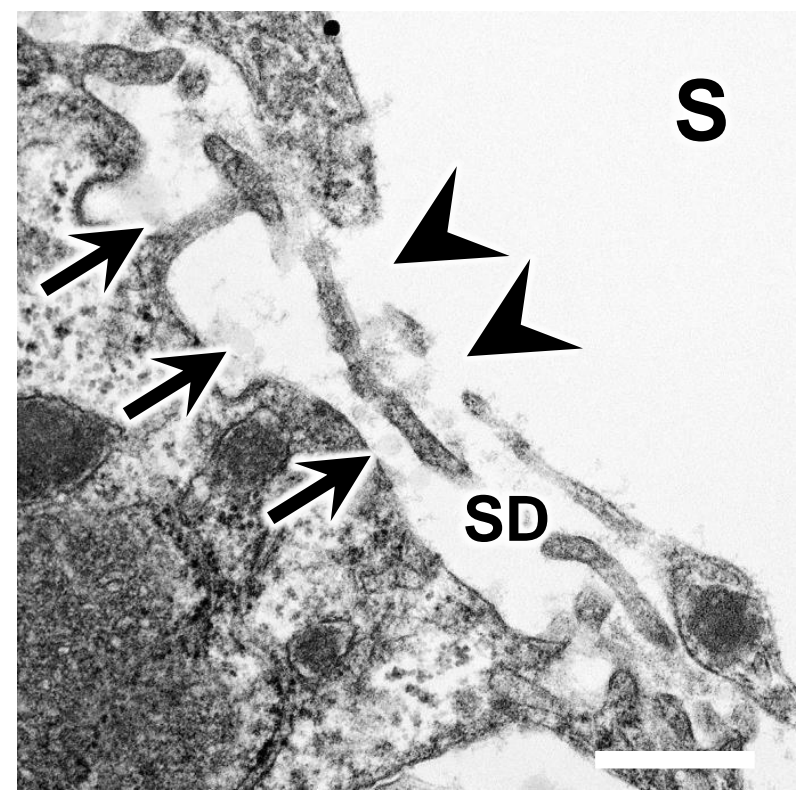

HFD 22 w

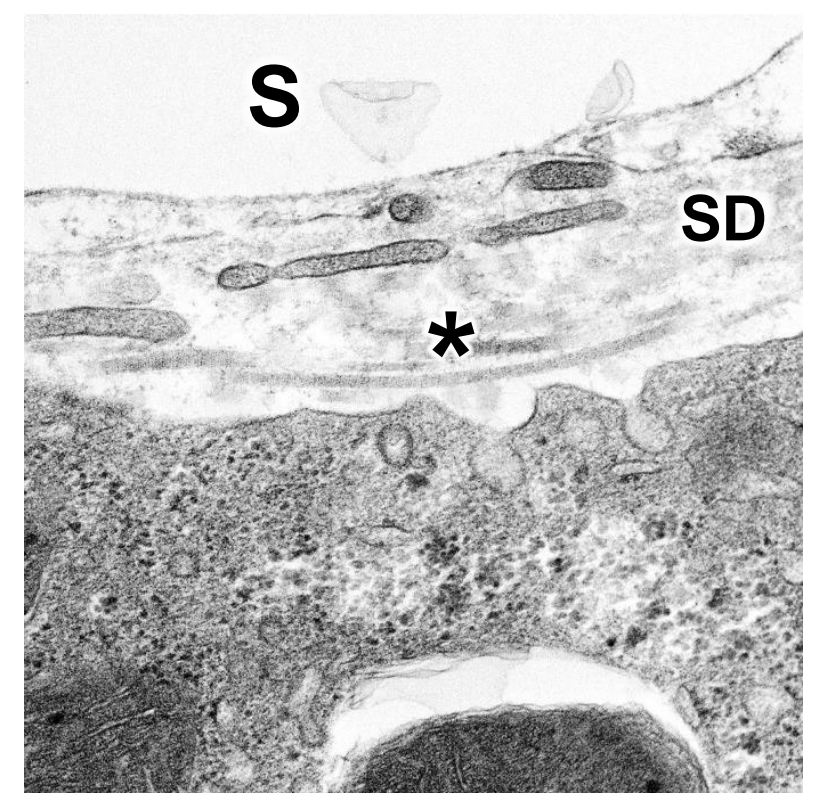

Supplementary Figure 5: Transmission electron microscopic images of livers of high fat diet (HFD) 8-week and HFD 22-week mice. Arrowheads indicate fenestrae. Arrows indicate small lipid particles. The asterisk indicates collagen deposits in the space of Disse. Bar = $500 \mathrm{~nm}$. S, sinusoid lumen; SD, space of Disse. 


\section{Supplementary Figure 6}

CDAA $4 \mathrm{w}$

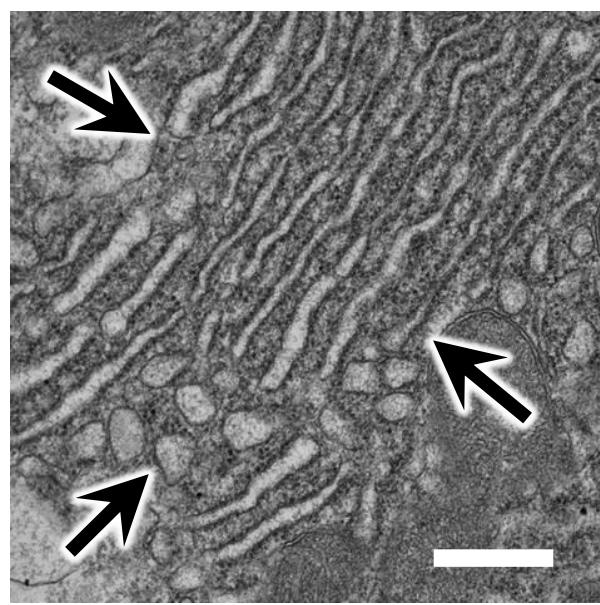

CDAA 22 w

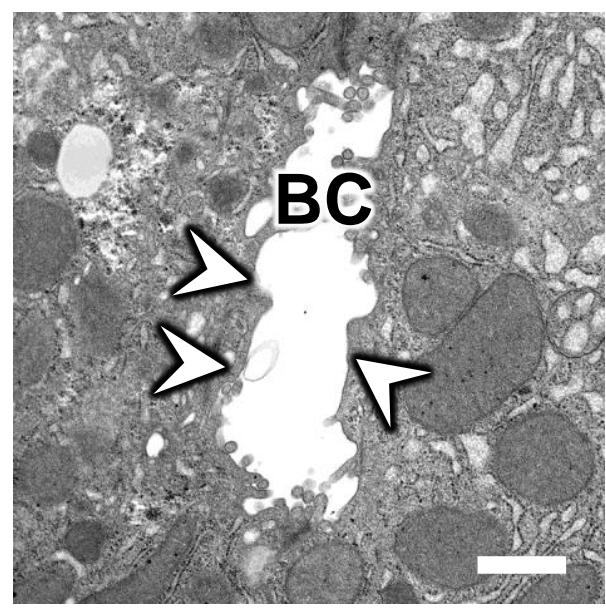

CDAA 8 w

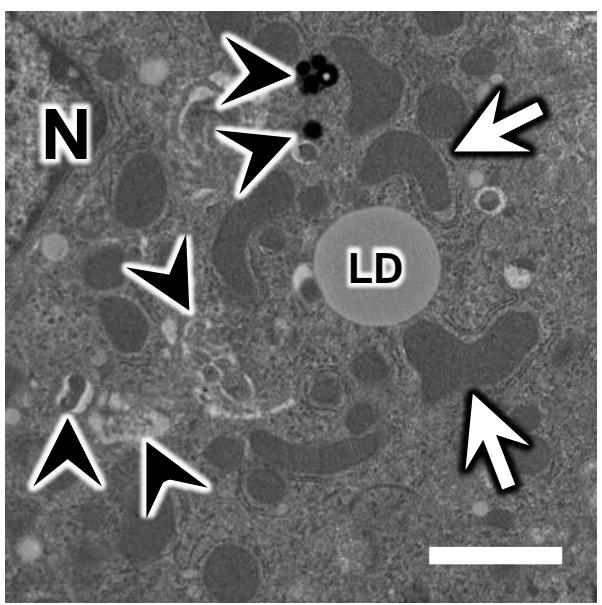

Recovery 4 w
CDAA 22 w

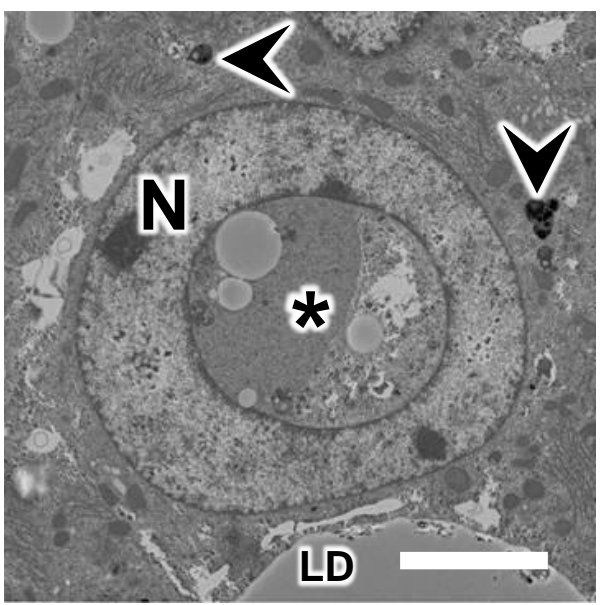

Supplementary Figure 6: Transmission electron microscopic images of livers in choline-deficient, L-amino acid-defined (CDAA) 4-week (bar $=500 \mathrm{~nm})$, CDAA 8week (bar $=2 \mu \mathrm{m}$ ), CDAA 22-week (top right panel: bar $=5 \mu \mathrm{m}$, bottom panel: bar $=1 \mu \mathrm{m}$ ), and recovery 4-week (8 weeks of CDAA feeding followed by an additional 4 weeks of standard diet feeding: bar $=1 \mu \mathrm{m}$ ) mice. Black arrows indicate enlarged endoplasmic reticulum. Black arrowheads indicate autophagosomes and lysosomes (high-density organelles). White arrowheads indicate loss of microvilli in the bile canaliculus. White arrows indicate deformed mitochondria. The asterisk indicates a pseudoinclusion body. N, nucleus of hepatocyte; LD, lipid droplet; BC, bile canaliculus. 


\section{Supplementary Figure 7}
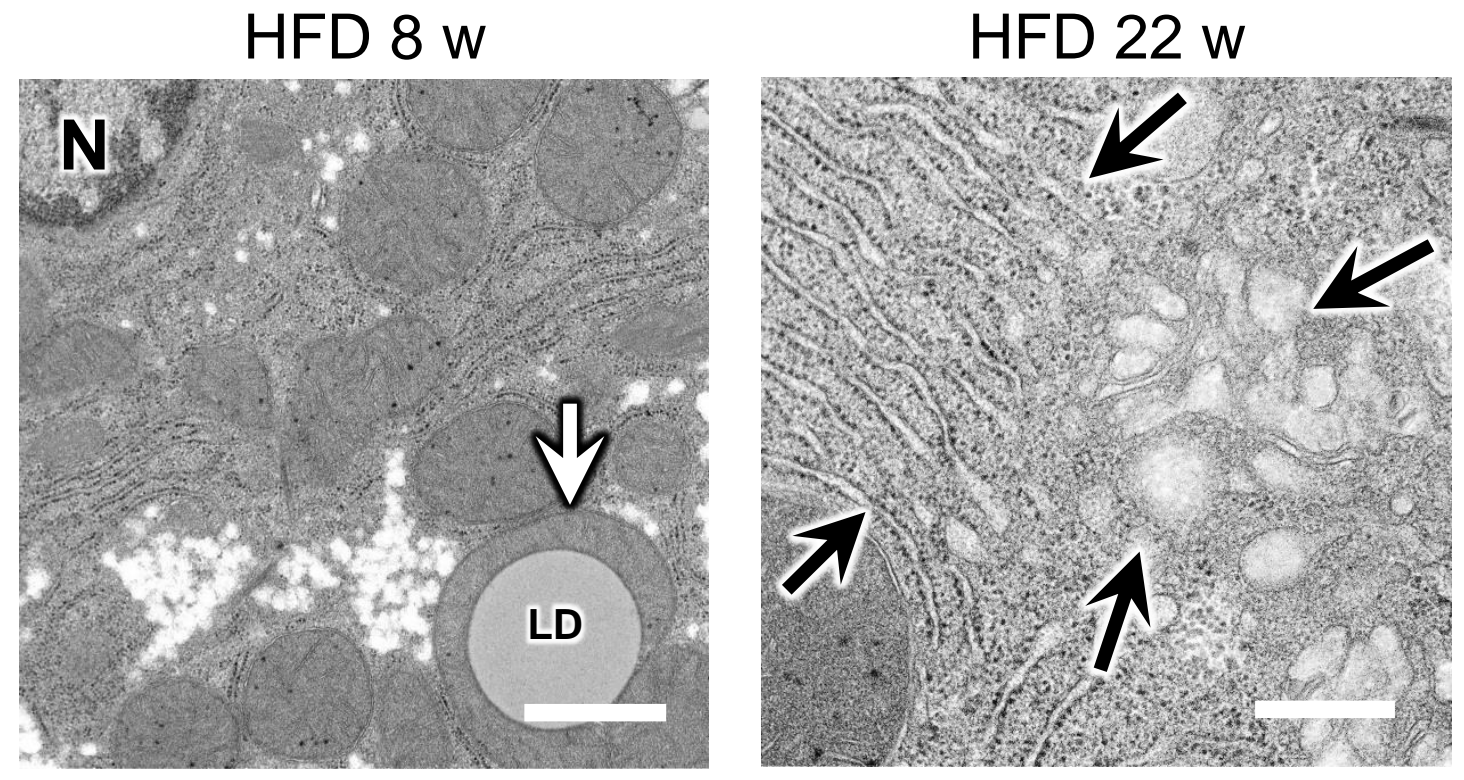

HFD 22 w

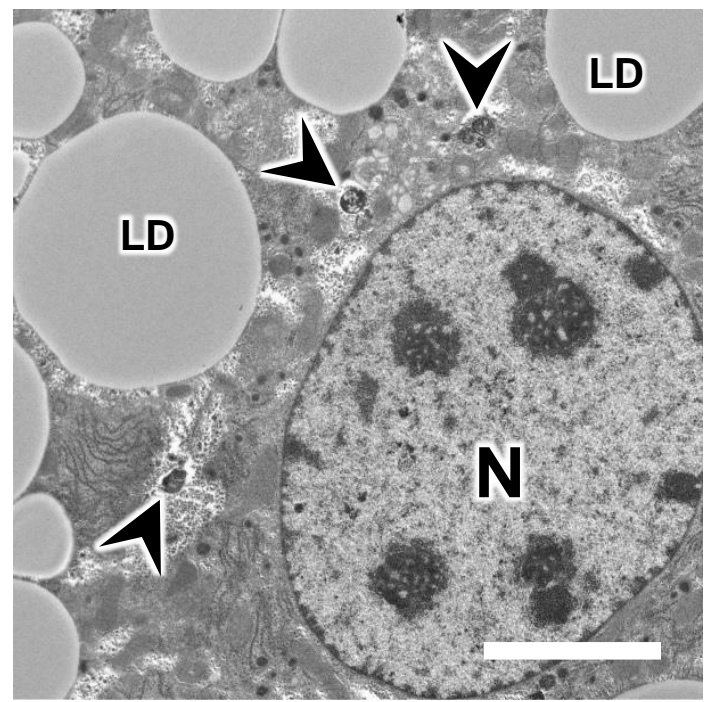

HFD 22 w

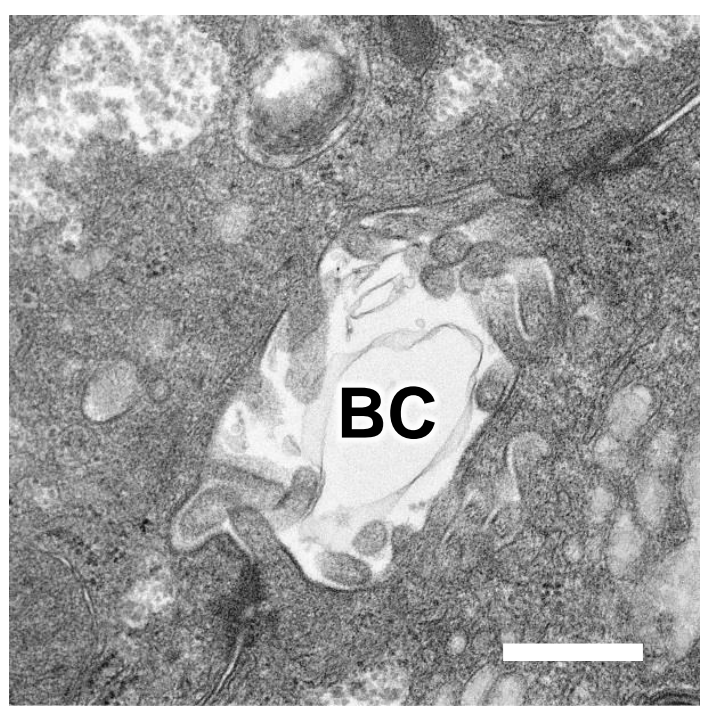

Supplementary Figure 7: Transmission electron microscopic images of livers in high fat diet (HFD) 8-week (bar $=1 \mu \mathrm{m}$ ) and HFD 22-week (top right panel: bar $=500 \mathrm{~nm}$, bottom left panel: bar $=5 \mu \mathrm{m}$, bottom right panel: bar $=500 \mathrm{~nm}$ ) mice. A white arrow indicates a deformed mitochondrion. Black arrows indicate enlarged endoplasmic reticula. Black arrowheads indicate autophagosomes and lysosomes (high-density organelles). Hepatocyte nuclei and a bile canaliculus show normal appearance. N, nucleus of hepatocyte; LD, lipid droplet; BC, bile canaliculus. 